\title{
Synthesis of Bridged Oxo Tungsten Complexes
}

Junes Ipaktschi,*'Parham Rooshenas, Thomas Klotzbach, Ansgar

Dülmer and Elmira Hüseynova

Institute of Organic Chemistry, Justus-Liebig University, Heinrich-Buff-Ring

58, D-35392 Giessen, Germany, Azerbaijan State Oil Academy, Baku, Azerbaijan

Received $x x \times x, 2004$

\section{SUPPLEMENTARY MATERIAL}

*To whom correspondence should be addressed

FAX: $+49 / 641 / 9936309$

E-mail: junes.ipaktschi@ org.chemie.uni-giessen.de 
Figure 1. Molecular structure and atom-numbering scheme for complex 4a without $\mathrm{H}$ atoms on $\mathrm{Cp}$ rings and phenyl groups. Thermal ellipsoids are shown at the $30 \%$ probability level.

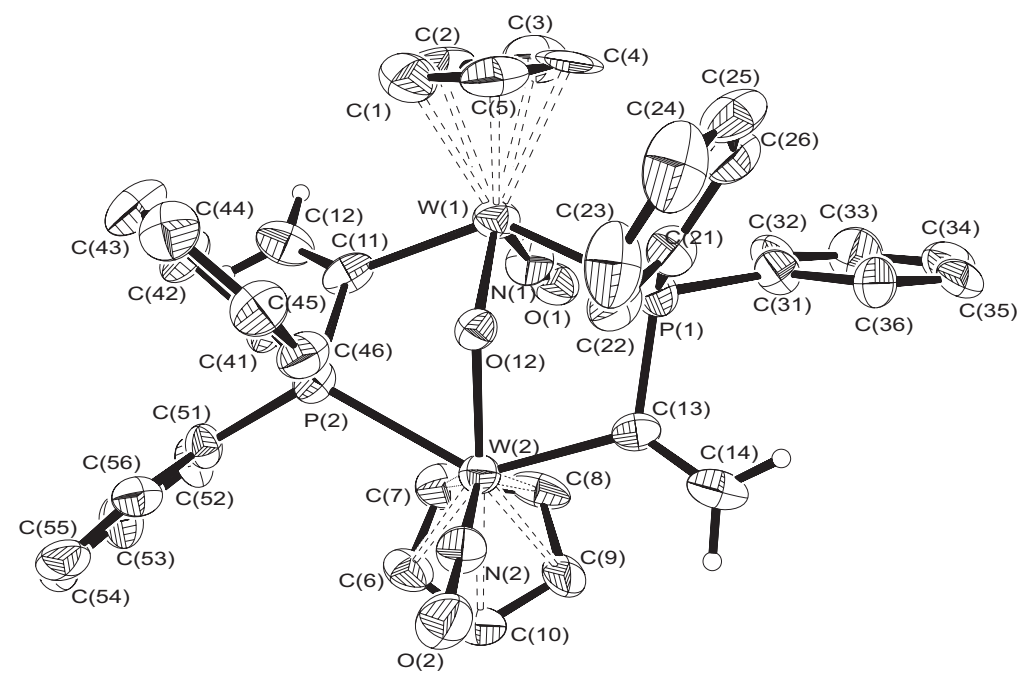

Table 1 Crystal data and structure refinement for $\mathbf{4 a}$.

Identification code 
Empirical formula

Formula weight

Temperature

Wavelength

Crystal system

Space group

Unit cell dimensions

Volume

Z

Density (calculated)

Absorption coefficient

$\mathrm{F}(000)$

Crystal size

Theta range for data collection

Index ranges

Reflections collected

Independent reflections

Completeness to theta $=20.93^{\circ}$

Refinement method

Data / restraints / parameters

Goodness-of-fit on $\mathrm{F}^{2}$

Final R indices [I>2sigma(I)]

$\mathrm{R}$ indices (all data)

Largest diff. peak and hole

\section{C155 H142 Cl6 N8 O12 P8 W8}

4240.03

293(2) K

$0.71073 \AA$

monoclinic

$\mathrm{C} 2 / \mathrm{c}$ (no 15)

$\mathrm{a}=44.354(9) \AA \quad \alpha=90^{\circ}$.

$\mathrm{b}=9.976(2) \AA \quad \beta=98.01(3)^{\circ}$.

$\mathrm{c}=34.402(7) \AA \quad \gamma=90^{\circ}$.

15074(5) $\AA^{3}$

4

$1.868 \mathrm{Mg} / \mathrm{m}^{3}$

$6.331 \mathrm{~mm}^{-1}$

8152

$0.08 \times 0.04 \times 0.27 \mathrm{~mm}^{3}$

1.61 to $20.93^{\circ}$.

$-44<=\mathrm{h}<=43,-9<=\mathrm{k}<=9,-34<=1<=34$

27231

$7846[\mathrm{R}(\mathrm{int})=0.0775]$

$98.1 \%$

Full-matrix least-squares on $\mathrm{F}^{2}$

7846 / 0 / 893

0.885

$\mathrm{R} 1=0.0381, \mathrm{wR} 2=0.0682$

$\mathrm{R} 1=0.0758, \mathrm{wR} 2=0.0754$

0.793 and -0.672 e. $\AA^{-3}$ 
Table 2. Atomic coordinates ( $\times 10^{4}$ ) and equivalent isotropic displacement parameters $\left(\AA^{2} \times 10^{3}\right)$ for $4 \mathrm{a}$. $\mathrm{U}(\mathrm{eq})$ is defined as one third of the trace of the orthogonalized $\mathrm{U}^{\mathrm{ij}}$ tensor.

\begin{tabular}{|c|c|c|c|c|}
\hline & $\mathrm{x}$ & $\mathrm{y}$ & $\mathrm{z}$ & $\mathrm{U}(\mathrm{eq})$ \\
\hline $\mathrm{W}(1)$ & $764(1)$ & $-5460(1)$ & $6735(1)$ & $60(1)$ \\
\hline $\mathrm{O}(1)$ & $1029(2)$ & $-2956(14)$ & $6479(3)$ & $84(3)$ \\
\hline $\mathrm{N}(1)$ & $921(3)$ & $-4031(15)$ & $6573(3)$ & $73(4)$ \\
\hline $\mathrm{P}(1)$ & $1282(1)$ & $-6302(4)$ & $7053(1)$ & $59(1)$ \\
\hline $\mathrm{W}(2)$ & 1189(1) & $-7031(1)$ & $6096(1)$ & $51(1)$ \\
\hline $\mathrm{O}(2)$ & $1343(2)$ & $-9803(13)$ & 5914(3) & $89(4)$ \\
\hline $\mathrm{N}(2)$ & $1269(3)$ & $-8699(14)$ & $6023(3)$ & $66(4)$ \\
\hline $\mathrm{P}(2)$ & $623(1)$ & $-7189(4)$ & $5908(1)$ & $53(1)$ \\
\hline $\mathrm{O}(12)$ & $919(2)$ & $-7114(10)$ & $6519(2)$ & $56(2)$ \\
\hline $\mathrm{C}(1)$ & $303(6)$ & $-6010(40)$ & $6944(6)$ & $116(8)$ \\
\hline $\mathrm{C}(2)$ & $329(6)$ & $-4620(40)$ & $6942(7)$ & $119(10)$ \\
\hline$C(3)$ & $558(7)$ & $-4270(30)$ & $7219(8)$ & $110(8)$ \\
\hline $\mathrm{C}(4)$ & $660(6)$ & $-5400(50)$ & 7394(5) & $128(11)$ \\
\hline $\mathrm{C}(5)$ & $517(6)$ & $-6490(30)$ & 7228(9) & $96(7)$ \\
\hline$C(6)$ & $1200(4)$ & $-6131(19)$ & $5474(4)$ & $71(5)$ \\
\hline$C(7)$ & $1130(4)$ & $-5096(19)$ & $5712(5)$ & $78(5)$ \\
\hline $\mathrm{C}(8)$ & $1386(5)$ & $-4893(16)$ & $5980(5)$ & $77(5)$ \\
\hline $\mathrm{C}(9)$ & 1599(4) & $-5850(20)$ & $5899(4)$ & $78(6)$ \\
\hline $\mathrm{C}(10)$ & 1481(4) & $-6593(17)$ & $5580(4)$ & $71(5)$ \\
\hline $\mathrm{C}(11)$ & 497(3) & $-5729(15)$ & $6163(4)$ & $58(4)$ \\
\hline $\mathrm{C}(12)$ & $268(4)$ & $-4998(17)$ & $5983(4)$ & $85(6)$ \\
\hline $\mathrm{C}(13)$ & 1483(3) & $-6837(15)$ & $6650(4)$ & $61(4)$ \\
\hline $\mathrm{C}(14)$ & $1770(4)$ & $-7177(17)$ & $6748(4)$ & $83(5)$ \\
\hline $\mathrm{C}(21)$ & $1229(3)$ & $-7831(19)$ & $7338(4)$ & $66(4)$ \\
\hline $\mathrm{C}(22)$ & 1183(3) & $-9010(20)$ & $7152(5)$ & $72(5)$ \\
\hline $\mathrm{C}(23)$ & $1110(4)$ & $-10174(19)$ & $7350(6)$ & $101(7)$ \\
\hline$C(24)$ & $1085(5)$ & $-10120(30)$ & $7740(8)$ & $119(8)$ \\
\hline$C(25)$ & 1131(4) & $-8940(30)$ & $7927(5)$ & $90(6)$ \\
\hline$C(26)$ & 1207(3) & $-7751(19)$ & $7739(5)$ & $78(5)$ \\
\hline $\mathrm{C}(31)$ & 1543(3) & $-5223(18)$ & $7368(4)$ & $60(4)$ \\
\hline$C(32)$ & $1526(4)$ & $-3870(20)$ & $7300(4)$ & $75(5)$ \\
\hline $\mathrm{C}(33)$ & $1742(5)$ & $-2990(20)$ & $7489(6)$ & $97(6)$ \\
\hline $\mathrm{C}(34)$ & 1978(5) & $-3440(20)$ & $7762(5)$ & $89(6)$ \\
\hline $\mathrm{C}(35)$ & 1995(4) & $-4800(30)$ & 7834(4) & $82(6)$ \\
\hline
\end{tabular}




\begin{tabular}{|c|c|c|c|c|}
\hline$C(36)$ & $1783(4)$ & $-5670(20)$ & 7643(4) & $79(5)$ \\
\hline $\mathrm{C}(41)$ & $434(3)$ & $-8577(15)$ & $6097(4)$ & $55(4)$ \\
\hline $\mathrm{C}(42)$ & $128(3)$ & $-8586(17)$ & $6076(4)$ & $68(4)$ \\
\hline $\mathrm{C}(43)$ & $-32(4)$ & $-9650(20)$ & $6209(5)$ & $90(5)$ \\
\hline $\mathrm{C}(44)$ & $115(4)$ & $-10752(18)$ & $6370(4)$ & $77(5)$ \\
\hline $\mathrm{C}(45)$ & $429(4)$ & $-10783(17)$ & 6401(4) & $72(5)$ \\
\hline $\mathrm{C}(46)$ & $583(3)$ & $-9706(18)$ & $6260(4)$ & $66(4)$ \\
\hline $\mathrm{C}(51)$ & $468(3)$ & $-7240(17)$ & $5387(4)$ & $51(4)$ \\
\hline $\mathrm{C}(52)$ & $455(3)$ & $-6123(17)$ & $5149(4)$ & $65(4)$ \\
\hline $\mathrm{C}(53)$ & $378(4)$ & $-6250(20)$ & $4744(4)$ & $79(5)$ \\
\hline $\mathrm{C}(54)$ & $329(3)$ & $-7440(20)$ & 4573(4) & $67(5)$ \\
\hline$C(55)$ & $346(3)$ & $-8578(18)$ & $4807(5)$ & $74(5)$ \\
\hline $\mathrm{C}(56)$ & $413(3)$ & $-8458(17)$ & $5208(4)$ & $59(4)$ \\
\hline $\mathrm{W}(1 \mathrm{~B})$ & $1866(1)$ & $-2077(1)$ & $4257(1)$ & $46(1)$ \\
\hline $\mathrm{N}(1 \mathrm{~B})$ & 2018(2) & $-1371(12)$ & $4693(3)$ & $49(3)$ \\
\hline $\mathrm{O}(1 \mathrm{~B})$ & $2132(2)$ & $-921(11)$ & $5018(3)$ & $78(3)$ \\
\hline $\mathrm{P}(1 \mathrm{~B})$ & 1993(1) & $107(4)$ & $3898(1)$ & $46(1)$ \\
\hline $\mathrm{W}(2 \mathrm{~B})$ & $1314(1)$ & $307(1)$ & $4209(1)$ & $42(1)$ \\
\hline $\mathrm{N}(2 \mathrm{~B})$ & $997(2)$ & $659(11)$ & $3860(3)$ & $46(3)$ \\
\hline $\mathrm{O}(2 \mathrm{~B})$ & $764(2)$ & $1102(11)$ & 3661(3) & $79(3)$ \\
\hline $\mathrm{P}(2 \mathrm{~B})$ & $1148(1)$ & $-1973(4)$ & $4429(1)$ & $42(1)$ \\
\hline $\mathrm{O}(12 \mathrm{~B})$ & $1492(2)$ & $-1296(9)$ & 3972(2) & $45(2)$ \\
\hline$C(11 B)$ & 1515(3) & $-2763(15)$ & $4583(3)$ & $57(4)$ \\
\hline$C(12 B)$ & $1537(3)$ & $-3570(17)$ & 4887(4) & $78(5)$ \\
\hline$C(13 B)$ & $1632(3)$ & $1004(15)$ & $3846(4)$ & $57(4)$ \\
\hline$C(14 B)$ & 1581(3) & 1917(17) & $3550(4)$ & $69(5)$ \\
\hline $\mathrm{C}(1 \mathrm{~B})$ & 1967(8) & $-4318(19)$ & $4252(9)$ & $93(7)$ \\
\hline $\mathrm{C}(2 \mathrm{~B})$ & $1841(5)$ & $-4080(30)$ & $3890(12)$ & $131(11)$ \\
\hline $\mathrm{C}(3 \mathrm{~B})$ & $2027(10)$ & $-3240(30)$ & $3761(7)$ & $127(11)$ \\
\hline $\mathrm{C}(4 \mathrm{~B})$ & $2265(6)$ & $-3090(20)$ & $3985(17)$ & $164(16)$ \\
\hline $\mathrm{C}(5 \mathrm{~B})$ & 2222(9) & $-3720(30)$ & $4345(8)$ & $141(13)$ \\
\hline $\mathrm{C}(6 \mathrm{~B})$ & 1171(3) & $1045(15)$ & 4801(4) & $53(4)$ \\
\hline $\mathrm{C}(7 \mathrm{~B})$ & $1488(3)$ & $647(15)$ & 4893(3) & $50(4)$ \\
\hline $\mathrm{C}(8 \mathrm{~B})$ & $1640(3)$ & 1499(17) & $4697(4)$ & $59(4)$ \\
\hline $\mathrm{C}(9 \mathrm{~B})$ & $1449(4)$ & $2417(17)$ & 4483(4) & $66(5)$ \\
\hline $\mathrm{C}(10 \mathrm{~B})$ & $1159(4)$ & 2173(16) & $4567(4)$ & $63(4)$ \\
\hline$C(21 B)$ & $2126(3)$ & $-88(13)$ & $3416(4)$ & $45(4)$ \\
\hline$C(22 B)$ & $1916(3)$ & $-373(15)$ & $3084(4)$ & $58(4)$ \\
\hline$C(23 B)$ & 2009(4) & $-598(17)$ & 2724(4) & $74(5)$ \\
\hline
\end{tabular}




\begin{tabular}{|c|c|c|c|c|}
\hline$C(24 B)$ & $2309(5)$ & $-545(19)$ & 2692(4) & $87(6)$ \\
\hline$C(25 B)$ & $2516(4)$ & $-260(20)$ & $3007(5)$ & $102(7)$ \\
\hline$C(26 B)$ & $2422(3)$ & $-12(16)$ & $3371(4)$ & $76(5)$ \\
\hline$C(31 B)$ & $2290(3)$ & 1219(17) & $4135(4)$ & $48(4)$ \\
\hline$C(36 B)$ & $2321(4)$ & $2500(20)$ & $4002(6)$ & $96(6)$ \\
\hline$C(35 B)$ & $2552(4)$ & 3311(18) & $4175(7)$ & $115(8)$ \\
\hline $\mathrm{C}(34 \mathrm{~B})$ & $2753(4)$ & $2860(30)$ & $4458(6)$ & $98(7)$ \\
\hline$C(33 B)$ & $2728(4)$ & 1610(30) & $4607(5)$ & $102(8)$ \\
\hline $\mathrm{C}(32 \mathrm{~B})$ & $2498(3)$ & $806(18)$ & $4438(4)$ & $78(5)$ \\
\hline$C(41 B)$ & $952(3)$ & $-3019(17)$ & 4037(3) & $46(3)$ \\
\hline$C(42 B)$ & $765(3)$ & $-2468(16)$ & $3723(4)$ & $65(5)$ \\
\hline$C(43 B)$ & $609(4)$ & $-3270(20)$ & $3448(4)$ & $81(5)$ \\
\hline$C(44 B)$ & $634(4)$ & $-4620(20)$ & $3468(4)$ & $85(6)$ \\
\hline$C(45 B)$ & $809(4)$ & $-5187(18)$ & $3766(6)$ & $91(6)$ \\
\hline$C(46 B)$ & $976(3)$ & $-4398(19)$ & $4055(4)$ & $71(5)$ \\
\hline$C(51 B)$ & $902(3)$ & $-2188(13)$ & 4811(3) & $42(3)$ \\
\hline$C(52 B)$ & 1001(3) & $-1884(13)$ & 5201(4) & $48(4)$ \\
\hline$C(53 B)$ & $808(3)$ & $-1901(15)$ & $5480(4)$ & $55(4)$ \\
\hline$C(54 B)$ & $509(4)$ & $-2188(16)$ & $5380(4)$ & $63(4)$ \\
\hline$C(55 B)$ & $396(3)$ & $-2501(14)$ & 4996(5) & $61(4)$ \\
\hline$C(56 \mathrm{~B})$ & $596(3)$ & $-2484(13)$ & $4718(3)$ & $49(4)$ \\
\hline$C(15)$ & $1862(7)$ & 1790(40) & 1390(7) & $270(20)$ \\
\hline $\mathrm{Cl}(1)$ & $1907(2)$ & 2091(10) & $933(3)$ & $219(4)$ \\
\hline $\mathrm{Cl}(2)$ & $2116(2)$ & $997(13)$ & $1674(3)$ & $267(5)$ \\
\hline$C(16)$ & 264(9) & $370(50)$ & $2658(19)$ & $170(30)$ \\
\hline $\mathrm{Cl}(3)$ & 0 & $1740(30)$ & 2500 & $760(40)$ \\
\hline $\mathrm{Cl}(4)$ & 0 & $-690(30)$ & 2500 & $466(17)$ \\
\hline
\end{tabular}


Table 3. Bond lengths $[\AA]$ and angles $\left[{ }^{\circ}\right]$ for $\mathbf{4 a}$.

\begin{tabular}{|c|c|}
\hline $\mathrm{W}(1)-\mathrm{N}(1)$ & $1.713(13)$ \\
\hline $\mathrm{W}(1)-\mathrm{O}(12)$ & $1.973(9)$ \\
\hline $\mathrm{W}(1)-\mathrm{C}(11)$ & $2.169(15)$ \\
\hline $\mathrm{W}(1)-\mathrm{C}(2)$ & $2.304(17)$ \\
\hline $\mathrm{W}(1)-\mathrm{C}(1)$ & $2.32(2)$ \\
\hline $\mathrm{W}(1)-\mathrm{C}(3)$ & $2.333(19)$ \\
\hline $\mathrm{W}(1)-\mathrm{C}(5)$ & $2.375(19)$ \\
\hline $\mathrm{W}(1)-\mathrm{C}(4)$ & $2.375(16)$ \\
\hline $\mathrm{W}(1)-\mathrm{P}(1)$ & $2.545(4)$ \\
\hline $\mathrm{O}(1)-\mathrm{N}(1)$ & $1.235(14)$ \\
\hline $\mathrm{P}(1)-\mathrm{C}(31)$ & $1.823(16)$ \\
\hline $\mathrm{P}(1)-\mathrm{C}(13)$ & $1.831(13)$ \\
\hline $\mathrm{P}(1)-\mathrm{C}(21)$ & $1.845(17)$ \\
\hline $\mathrm{W}(2)-\mathrm{N}(2)$ & $1.727(13)$ \\
\hline $\mathrm{W}(2)-\mathrm{O}(12)$ & $2.011(8)$ \\
\hline $\mathrm{W}(2)-\mathrm{C}(13)$ & $2.162(14)$ \\
\hline $\mathrm{W}(2)-\mathrm{C}(6)$ & $2.328(13)$ \\
\hline $\mathrm{W}(2)-\mathrm{C}(7)$ & $2.333(15)$ \\
\hline $\mathrm{W}(2)-\mathrm{C}(9)$ & $2.348(14)$ \\
\hline $\mathrm{W}(2)-\mathrm{C}(8)$ & $2.360(15)$ \\
\hline $\mathrm{W}(2)-\mathrm{C}(10)$ & $2.378(14)$ \\
\hline $\mathrm{W}(2)-\mathrm{P}(2)$ & $2.508(4)$ \\
\hline $\mathrm{O}(2)-\mathrm{N}(2)$ & $1.223(14)$ \\
\hline $\mathrm{P}(2)-\mathrm{C}(41)$ & $1.788(15)$ \\
\hline $\mathrm{P}(2)-\mathrm{C}(51)$ & $1.827(12)$ \\
\hline $\mathrm{P}(2)-\mathrm{C}(11)$ & $1.828(15)$ \\
\hline$C(1)-C(5)$ & $1.35(3)$ \\
\hline $\mathrm{C}(1)-\mathrm{C}(2)$ & $1.39(3)$ \\
\hline $\mathrm{C}(2)-\mathrm{C}(3)$ & $1.34(3)$ \\
\hline$C(3)-C(4)$ & $1.33(3)$ \\
\hline$C(4)-C(5)$ & $1.34(3)$ \\
\hline$C(6)-C(10)$ & $1.329(19)$ \\
\hline$C(6)-C(7)$ & $1.38(2)$ \\
\hline $\mathrm{C}(7)-\mathrm{C}(8)$ & $1.38(2)$ \\
\hline $\mathrm{C}(8)-\mathrm{C}(9)$ & $1.40(2)$ \\
\hline$C(9)-C(10)$ & $1.368(19)$ \\
\hline $\mathrm{C}(11)-\mathrm{C}(12)$ & $1.331(18)$ \\
\hline
\end{tabular}




\begin{tabular}{|c|c|}
\hline $\mathrm{C}(13)-\mathrm{C}(14)$ & $1.314(18)$ \\
\hline$C(21)-C(22)$ & $1.34(2)$ \\
\hline$C(21)-C(26)$ & $1.399(18)$ \\
\hline$C(22)-C(23)$ & $1.41(2)$ \\
\hline$C(23)-C(24)$ & $1.36(3)$ \\
\hline$C(24)-C(25)$ & $1.34(3)$ \\
\hline$C(25)-C(26)$ & $1.42(2)$ \\
\hline $\mathrm{C}(31)-\mathrm{C}(32)$ & $1.37(2)$ \\
\hline $\mathrm{C}(31)-\mathrm{C}(36)$ & $1.393(18)$ \\
\hline $\mathrm{C}(32)-\mathrm{C}(33)$ & $1.39(2)$ \\
\hline $\mathrm{C}(33)-\mathrm{C}(34)$ & $1.38(2)$ \\
\hline $\mathrm{C}(34)-\mathrm{C}(35)$ & $1.37(2)$ \\
\hline$C(35)-C(36)$ & $1.38(2)$ \\
\hline $\mathrm{C}(41)-\mathrm{C}(42)$ & $1.351(18)$ \\
\hline $\mathrm{C}(41)-\mathrm{C}(46)$ & $1.383(19)$ \\
\hline $\mathrm{C}(42)-\mathrm{C}(43)$ & $1.39(2)$ \\
\hline $\mathrm{C}(43)-\mathrm{C}(44)$ & $1.36(2)$ \\
\hline $\mathrm{C}(44)-\mathrm{C}(45)$ & $1.38(2)$ \\
\hline$C(45)-C(46)$ & $1.395(19)$ \\
\hline $\mathrm{C}(51)-\mathrm{C}(56)$ & $1.369(19)$ \\
\hline $\mathrm{C}(51)-\mathrm{C}(52)$ & $1.380(19)$ \\
\hline $\mathrm{C}(52)-\mathrm{C}(53)$ & $1.392(18)$ \\
\hline $\mathrm{C}(53)-\mathrm{C}(54)$ & $1.33(2)$ \\
\hline $\mathrm{C}(54)-\mathrm{C}(55)$ & $1.39(2)$ \\
\hline $\mathrm{C}(55)-\mathrm{C}(56)$ & $1.375(18)$ \\
\hline W(1B)-N(1B) & $1.711(11)$ \\
\hline $\mathrm{W}(1 \mathrm{~B})-\mathrm{O}(12 \mathrm{~B})$ & $1.965(7)$ \\
\hline $\mathrm{W}(1 \mathrm{~B})-\mathrm{C}(11 \mathrm{~B})$ & $2.152(14)$ \\
\hline $\mathrm{W}(1 \mathrm{~B})-\mathrm{C}(3 \mathrm{~B})$ & $2.26(2)$ \\
\hline $\mathrm{W}(1 \mathrm{~B})-\mathrm{C}(5 \mathrm{~B})$ & $2.27(2)$ \\
\hline $\mathrm{W}(1 \mathrm{~B})-\mathrm{C}(1 \mathrm{~B})$ & $2.281(17)$ \\
\hline W(1B)-C(4B) & $2.345(16)$ \\
\hline $\mathrm{W}(1 \mathrm{~B})-\mathrm{C}(2 \mathrm{~B})$ & $2.36(2)$ \\
\hline $\mathrm{W}(1 \mathrm{~B})-\mathrm{P}(1 \mathrm{~B})$ & $2.604(4)$ \\
\hline $\mathrm{N}(1 \mathrm{~B})-\mathrm{O}(1 \mathrm{~B})$ & $1.244(11)$ \\
\hline $\mathrm{P}(1 \mathrm{~B})-\mathrm{C}(31 \mathrm{~B})$ & $1.823(14)$ \\
\hline $\mathrm{P}(1 \mathrm{~B})-\mathrm{C}(13 \mathrm{~B})$ & $1.825(14)$ \\
\hline $\mathrm{P}(1 \mathrm{~B})-\mathrm{C}(21 \mathrm{~B})$ & $1.845(12)$ \\
\hline $\mathrm{W}(2 \mathrm{~B})-\mathrm{N}(2 \mathrm{~B})$ & $1.753(11)$ \\
\hline
\end{tabular}




\begin{tabular}{|c|c|}
\hline $\mathrm{W}(2 \mathrm{~B})-\mathrm{O}(12 \mathrm{~B})$ & $2.006(8)$ \\
\hline $\mathrm{W}(2 \mathrm{~B})-\mathrm{C}(13 \mathrm{~B})$ & $2.126(15)$ \\
\hline $\mathrm{W}(2 \mathrm{~B})-\mathrm{C}(6 \mathrm{~B})$ & $2.336(12)$ \\
\hline $\mathrm{W}(2 \mathrm{~B})-\mathrm{C}(9 \mathrm{~B})$ & $2.350(16)$ \\
\hline $\mathrm{W}(2 \mathrm{~B})-\mathrm{C}(8 \mathrm{~B})$ & $2.375(13)$ \\
\hline $\mathrm{W}(2 \mathrm{~B})-\mathrm{C}(10 \mathrm{~B})$ & $2.386(14)$ \\
\hline $\mathrm{W}(2 \mathrm{~B})-\mathrm{C}(7 \mathrm{~B})$ & $2.397(11)$ \\
\hline $\mathrm{W}(2 \mathrm{~B})-\mathrm{P}(2 \mathrm{~B})$ & $2.538(4)$ \\
\hline $\mathrm{N}(2 \mathrm{~B})-\mathrm{O}(2 \mathrm{~B})$ & $1.238(12)$ \\
\hline $\mathrm{P}(2 \mathrm{~B})-\mathrm{C}(11 \mathrm{~B})$ & $1.821(13)$ \\
\hline $\mathrm{P}(2 \mathrm{~B})-\mathrm{C}(41 \mathrm{~B})$ & $1.827(13)$ \\
\hline $\mathrm{P}(2 \mathrm{~B})-\mathrm{C}(51 \mathrm{~B})$ & $1.834(12)$ \\
\hline $\mathrm{C}(11 \mathrm{~B})-\mathrm{C}(12 \mathrm{~B})$ & $1.313(18)$ \\
\hline $\mathrm{C}(13 \mathrm{~B})-\mathrm{C}(14 \mathrm{~B})$ & $1.360(18)$ \\
\hline $\mathrm{C}(1 \mathrm{~B})-\mathrm{C}(5 \mathrm{~B})$ & $1.28(3)$ \\
\hline $\mathrm{C}(1 \mathrm{~B})-\mathrm{C}(2 \mathrm{~B})$ & $1.31(3)$ \\
\hline $\mathrm{C}(2 \mathrm{~B})-\mathrm{C}(3 \mathrm{~B})$ & $1.30(3)$ \\
\hline $\mathrm{C}(3 \mathrm{~B})-\mathrm{C}(4 \mathrm{~B})$ & $1.23(4)$ \\
\hline $\mathrm{C}(4 \mathrm{~B})-\mathrm{C}(5 \mathrm{~B})$ & $1.42(3)$ \\
\hline$C(6 B)-C(10 B)$ & $1.380(18)$ \\
\hline $\mathrm{C}(6 \mathrm{~B})-\mathrm{C}(7 \mathrm{~B})$ & $1.452(17)$ \\
\hline $\mathrm{C}(7 \mathrm{~B})-\mathrm{C}(8 \mathrm{~B})$ & $1.326(17)$ \\
\hline $\mathrm{C}(8 \mathrm{~B})-\mathrm{C}(9 \mathrm{~B})$ & $1.387(19)$ \\
\hline$C(9 B)-C(10 B)$ & $1.379(18)$ \\
\hline $\mathrm{C}(21 \mathrm{~B})-\mathrm{C}(26 \mathrm{~B})$ & $1.345(17)$ \\
\hline $\mathrm{C}(21 \mathrm{~B})-\mathrm{C}(22 \mathrm{~B})$ & $1.399(16)$ \\
\hline $\mathrm{C}(22 \mathrm{~B})-\mathrm{C}(23 \mathrm{~B})$ & $1.377(17)$ \\
\hline $\mathrm{C}(23 \mathrm{~B})-\mathrm{C}(24 \mathrm{~B})$ & $1.35(2)$ \\
\hline $\mathrm{C}(24 \mathrm{~B})-\mathrm{C}(25 \mathrm{~B})$ & $1.35(2)$ \\
\hline $\mathrm{C}(25 \mathrm{~B})-\mathrm{C}(26 \mathrm{~B})$ & $1.395(19)$ \\
\hline $\mathrm{C}(31 \mathrm{~B})-\mathrm{C}(32 \mathrm{~B})$ & $1.359(18)$ \\
\hline $\mathrm{C}(31 \mathrm{~B})-\mathrm{C}(36 \mathrm{~B})$ & $1.37(2)$ \\
\hline $\mathrm{C}(36 \mathrm{~B})-\mathrm{C}(35 \mathrm{~B})$ & $1.37(2)$ \\
\hline $\mathrm{C}(35 \mathrm{~B})-\mathrm{C}(34 \mathrm{~B})$ & $1.31(2)$ \\
\hline $\mathrm{C}(34 \mathrm{~B})-\mathrm{C}(33 \mathrm{~B})$ & $1.36(3)$ \\
\hline $\mathrm{C}(33 \mathrm{~B})-\mathrm{C}(32 \mathrm{~B})$ & $1.36(2)$ \\
\hline $\mathrm{C}(41 \mathrm{~B})-\mathrm{C}(42 \mathrm{~B})$ & $1.381(17)$ \\
\hline$C(41 B)-C(46 B)$ & $1.381(19)$ \\
\hline $\mathrm{C}(42 \mathrm{~B})-\mathrm{C}(43 \mathrm{~B})$ & $1.353(19)$ \\
\hline
\end{tabular}




\begin{tabular}{|c|c|}
\hline $\mathrm{C}(43 \mathrm{~B})-\mathrm{C}(44 \mathrm{~B})$ & $1.35(2)$ \\
\hline $\mathrm{C}(44 \mathrm{~B})-\mathrm{C}(45 \mathrm{~B})$ & $1.32(2)$ \\
\hline $\mathrm{C}(45 \mathrm{~B})-\mathrm{C}(46 \mathrm{~B})$ & $1.40(2)$ \\
\hline $\mathrm{C}(51 \mathrm{~B})-\mathrm{C}(56 \mathrm{~B})$ & $1.384(15)$ \\
\hline $\mathrm{C}(51 \mathrm{~B})-\mathrm{C}(52 \mathrm{~B})$ & $1.387(15)$ \\
\hline $\mathrm{C}(52 \mathrm{~B})-\mathrm{C}(53 \mathrm{~B})$ & $1.372(16)$ \\
\hline $\mathrm{C}(53 \mathrm{~B})-\mathrm{C}(54 \mathrm{~B})$ & $1.353(18)$ \\
\hline $\mathrm{C}(54 \mathrm{~B})-\mathrm{C}(55 \mathrm{~B})$ & $1.381(17)$ \\
\hline $\mathrm{C}(55 \mathrm{~B})-\mathrm{C}(56 \mathrm{~B})$ & $1.392(16)$ \\
\hline $\mathrm{C}(15)-\mathrm{Cl}(2)$ & $1.59(2)$ \\
\hline $\mathrm{C}(15)-\mathrm{Cl}(1)$ & $1.64(2)$ \\
\hline $\mathrm{C}(16)-\mathrm{Cl}(4)$ & $1.62(4)$ \\
\hline $\mathrm{C}(16)-\mathrm{Cl}(3)$ & $1.83(5)$ \\
\hline $\mathrm{Cl}(3)-\mathrm{C}(16) \# 1$ & $1.83(5)$ \\
\hline $\mathrm{Cl}(3)-\mathrm{Cl}(4)$ & $2.42(4)$ \\
\hline $\mathrm{Cl}(4)-\mathrm{C}(16) \# 1$ & $1.62(4)$ \\
\hline $\mathrm{N}(1)-\mathrm{W}(1)-\mathrm{O}(12)$ & $113.2(5)$ \\
\hline $\mathrm{N}(1)-\mathrm{W}(1)-\mathrm{C}(11)$ & $90.1(5)$ \\
\hline $\mathrm{O}(12)-\mathrm{W}(1)-\mathrm{C}(11)$ & $74.2(4)$ \\
\hline $\mathrm{N}(1)-\mathrm{W}(1)-\mathrm{C}(2)$ & $101.2(10)$ \\
\hline $\mathrm{O}(12)-\mathrm{W}(1)-\mathrm{C}(2)$ & $140.4(11)$ \\
\hline $\mathrm{C}(11)-\mathrm{W}(1)-\mathrm{C}(2)$ & 87.1(9) \\
\hline $\mathrm{N}(1)-\mathrm{W}(1)-\mathrm{C}(1)$ & $135.3(11)$ \\
\hline $\mathrm{O}(12)-\mathrm{W}(1)-\mathrm{C}(1)$ & 106.7(9) \\
\hline $\mathrm{C}(11)-\mathrm{W}(1)-\mathrm{C}(1)$ & $81.8(7)$ \\
\hline $\mathrm{C}(2)-\mathrm{W}(1)-\mathrm{C}(1)$ & $34.9(7)$ \\
\hline $\mathrm{N}(1)-\mathrm{W}(1)-\mathrm{C}(3)$ & $91.5(8)$ \\
\hline $\mathrm{O}(12)-\mathrm{W}(1)-\mathrm{C}(3)$ & $152.6(8)$ \\
\hline $\mathrm{C}(11)-\mathrm{W}(1)-\mathrm{C}(3)$ & $119.5(10)$ \\
\hline $\mathrm{C}(2)-\mathrm{W}(1)-\mathrm{C}(3)$ & $33.6(7)$ \\
\hline $\mathrm{C}(1)-\mathrm{W}(1)-\mathrm{C}(3)$ & $56.7(9)$ \\
\hline $\mathrm{N}(1)-\mathrm{W}(1)-\mathrm{C}(5)$ & 147.2(8) \\
\hline $\mathrm{O}(12)-\mathrm{W}(1)-\mathrm{C}(5)$ & $97.4(7)$ \\
\hline $\mathrm{C}(11)-\mathrm{W}(1)-\mathrm{C}(5)$ & 110.2(9) \\
\hline $\mathrm{C}(2)-\mathrm{W}(1)-\mathrm{C}(5)$ & $56.4(8)$ \\
\hline $\mathrm{C}(1)-\mathrm{W}(1)-\mathrm{C}(5)$ & $33.4(6)$ \\
\hline $\mathrm{C}(3)-\mathrm{W}(1)-\mathrm{C}(5)$ & $56.3(8)$ \\
\hline $\mathrm{N}(1)-\mathrm{W}(1)-\mathrm{C}(4)$ & $115.6(13)$ \\
\hline
\end{tabular}




\begin{tabular}{|c|c|}
\hline $\mathrm{O}(12)-\mathrm{W}(1)-\mathrm{C}(4)$ & $120.4(11)$ \\
\hline $\mathrm{C}(11)-\mathrm{W}(1)-\mathrm{C}(4)$ & $135.6(8)$ \\
\hline $\mathrm{C}(2)-\mathrm{W}(1)-\mathrm{C}(4)$ & $54.2(7)$ \\
\hline $\mathrm{C}(1)-\mathrm{W}(1)-\mathrm{C}(4)$ & $54.2(8)$ \\
\hline $\mathrm{C}(3)-\mathrm{W}(1)-\mathrm{C}(4)$ & $32.8(7)$ \\
\hline$C(5)-W(1)-C(4)$ & $32.9(8)$ \\
\hline $\mathrm{N}(1)-\mathrm{W}(1)-\mathrm{P}(1)$ & $91.8(4)$ \\
\hline $\mathrm{O}(12)-\mathrm{W}(1)-\mathrm{P}(1)$ & $63.0(2)$ \\
\hline $\mathrm{C}(11)-\mathrm{W}(1)-\mathrm{P}(1)$ & $134.0(4)$ \\
\hline $\mathrm{C}(2)-\mathrm{W}(1)-\mathrm{P}(1)$ & $137.1(7)$ \\
\hline $\mathrm{C}(1)-\mathrm{W}(1)-\mathrm{P}(1)$ & $124.7(9)$ \\
\hline $\mathrm{C}(3)-\mathrm{W}(1)-\mathrm{P}(1)$ & $106.4(9)$ \\
\hline $\mathrm{C}(5)-\mathrm{W}(1)-\mathrm{P}(1)$ & $91.7(7)$ \\
\hline $\mathrm{C}(4)-\mathrm{W}(1)-\mathrm{P}(1)$ & $83.2(6)$ \\
\hline $\mathrm{O}(1)-\mathrm{N}(1)-\mathrm{W}(1)$ & $175.5(12)$ \\
\hline $\mathrm{C}(31)-\mathrm{P}(1)-\mathrm{C}(13)$ & $106.6(7)$ \\
\hline $\mathrm{C}(31)-\mathrm{P}(1)-\mathrm{C}(21)$ & $106.9(7)$ \\
\hline $\mathrm{C}(13)-\mathrm{P}(1)-\mathrm{C}(21)$ & $105.9(8)$ \\
\hline $\mathrm{C}(31)-\mathrm{P}(1)-\mathrm{W}(1)$ & $121.4(5)$ \\
\hline $\mathrm{C}(13)-\mathrm{P}(1)-\mathrm{W}(1)$ & $106.0(5)$ \\
\hline $\mathrm{C}(21)-\mathrm{P}(1)-\mathrm{W}(1)$ & $109.1(5)$ \\
\hline $\mathrm{N}(2)-\mathrm{W}(2)-\mathrm{O}(12)$ & $102.7(5)$ \\
\hline $\mathrm{N}(2)-\mathrm{W}(2)-\mathrm{C}(13)$ & $95.9(5)$ \\
\hline $\mathrm{O}(12)-\mathrm{W}(2)-\mathrm{C}(13)$ & $73.3(4)$ \\
\hline $\mathrm{N}(2)-\mathrm{W}(2)-\mathrm{C}(6)$ & $102.0(6)$ \\
\hline $\mathrm{O}(12)-\mathrm{W}(2)-\mathrm{C}(6)$ & $140.6(6)$ \\
\hline $\mathrm{C}(13)-\mathrm{W}(2)-\mathrm{C}(6)$ & $133.5(6)$ \\
\hline $\mathrm{N}(2)-\mathrm{W}(2)-\mathrm{C}(7)$ & $136.5(6)$ \\
\hline $\mathrm{O}(12)-\mathrm{W}(2)-\mathrm{C}(7)$ & $114.3(5)$ \\
\hline $\mathrm{C}(13)-\mathrm{W}(2)-\mathrm{C}(7)$ & $115.5(7)$ \\
\hline $\mathrm{C}(6)-\mathrm{W}(2)-\mathrm{C}(7)$ & $34.5(5)$ \\
\hline $\mathrm{N}(2)-\mathrm{W}(2)-\mathrm{C}(9)$ & 105.1(7) \\
\hline $\mathrm{O}(12)-\mathrm{W}(2)-\mathrm{C}(9)$ & $142.5(5)$ \\
\hline $\mathrm{C}(13)-\mathrm{W}(2)-\mathrm{C}(9)$ & $79.2(5)$ \\
\hline $\mathrm{C}(6)-\mathrm{W}(2)-\mathrm{C}(9)$ & $54.8(5)$ \\
\hline $\mathrm{C}(7)-\mathrm{W}(2)-\mathrm{C}(9)$ & $56.7(6)$ \\
\hline $\mathrm{N}(2)-\mathrm{W}(2)-\mathrm{C}(8)$ & 139.3(6) \\
\hline $\mathrm{O}(12)-\mathrm{W}(2)-\mathrm{C}(8)$ & $115.5(5)$ \\
\hline $\mathrm{C}(13)-\mathrm{W}(2)-\mathrm{C}(8)$ & $82.8(6)$ \\
\hline
\end{tabular}




\begin{tabular}{|c|c|}
\hline $\mathrm{C}(6)-\mathrm{W}(2)-\mathrm{C}(8)$ & $56.0(5)$ \\
\hline $\mathrm{C}(7)-\mathrm{W}(2)-\mathrm{C}(8)$ & $34.1(5)$ \\
\hline $\mathrm{C}(9)-\mathrm{W}(2)-\mathrm{C}(8)$ & $34.5(5)$ \\
\hline $\mathrm{N}(2)-\mathrm{W}(2)-\mathrm{C}(10)$ & $85.7(6)$ \\
\hline $\mathrm{O}(12)-\mathrm{W}(2)-\mathrm{C}(10)$ & $171.3(5)$ \\
\hline $\mathrm{C}(13)-\mathrm{W}(2)-\mathrm{C}(10)$ & $108.5(6)$ \\
\hline $\mathrm{C}(6)-\mathrm{W}(2)-\mathrm{C}(10)$ & $32.8(5)$ \\
\hline$C(7)-W(2)-C(10)$ & $57.0(6)$ \\
\hline $\mathrm{C}(9)-\mathrm{W}(2)-\mathrm{C}(10)$ & $33.7(5)$ \\
\hline $\mathrm{C}(8)-\mathrm{W}(2)-\mathrm{C}(10)$ & $57.0(5)$ \\
\hline $\mathrm{N}(2)-\mathrm{W}(2)-\mathrm{P}(2)$ & $97.0(4)$ \\
\hline $\mathrm{O}(12)-\mathrm{W}(2)-\mathrm{P}(2)$ & $60.6(2)$ \\
\hline $\mathrm{C}(13)-\mathrm{W}(2)-\mathrm{P}(2)$ & $133.8(4)$ \\
\hline $\mathrm{C}(6)-\mathrm{W}(2)-\mathrm{P}(2)$ & $86.4(5)$ \\
\hline $\mathrm{C}(7)-\mathrm{W}(2)-\mathrm{P}(2)$ & $82.9(5)$ \\
\hline $\mathrm{C}(9)-\mathrm{W}(2)-\mathrm{P}(2)$ & $138.1(4)$ \\
\hline $\mathrm{C}(8)-\mathrm{W}(2)-\mathrm{P}(2)$ & $113.2(5)$ \\
\hline $\mathrm{C}(10)-\mathrm{W}(2)-\mathrm{P}(2)$ & $116.6(5)$ \\
\hline $\mathrm{O}(2)-\mathrm{N}(2)-\mathrm{W}(2)$ & $169.0(12)$ \\
\hline $\mathrm{C}(41)-\mathrm{P}(2)-\mathrm{C}(51)$ & $101.8(7)$ \\
\hline $\mathrm{C}(41)-\mathrm{P}(2)-\mathrm{C}(11)$ & $104.5(6)$ \\
\hline $\mathrm{C}(51)-\mathrm{P}(2)-\mathrm{C}(11)$ & $113.3(7)$ \\
\hline $\mathrm{C}(41)-\mathrm{P}(2)-\mathrm{W}(2)$ & $117.2(5)$ \\
\hline $\mathrm{C}(51)-\mathrm{P}(2)-\mathrm{W}(2)$ & $118.7(4)$ \\
\hline $\mathrm{C}(11)-\mathrm{P}(2)-\mathrm{W}(2)$ & $100.9(5)$ \\
\hline $\mathrm{W}(1)-\mathrm{O}(12)-\mathrm{W}(2)$ & $120.9(5)$ \\
\hline $\mathrm{C}(5)-\mathrm{C}(1)-\mathrm{C}(2)$ & $108(2)$ \\
\hline $\mathrm{C}(5)-\mathrm{C}(1)-\mathrm{W}(1)$ & $75.4(12)$ \\
\hline $\mathrm{C}(2)-\mathrm{C}(1)-\mathrm{W}(1)$ & $71.8(13)$ \\
\hline $\mathrm{C}(3)-\mathrm{C}(2)-\mathrm{C}(1)$ & $108(2)$ \\
\hline $\mathrm{C}(3)-\mathrm{C}(2)-\mathrm{W}(1)$ & 74.4(11) \\
\hline $\mathrm{C}(1)-\mathrm{C}(2)-\mathrm{W}(1)$ & $73.3(12)$ \\
\hline $\mathrm{C}(4)-\mathrm{C}(3)-\mathrm{C}(2)$ & $106(3)$ \\
\hline $\mathrm{C}(4)-\mathrm{C}(3)-\mathrm{W}(1)$ & $75.4(12)$ \\
\hline $\mathrm{C}(2)-\mathrm{C}(3)-\mathrm{W}(1)$ & $72.0(12)$ \\
\hline$C(3)-C(4)-C(5)$ & $112(2)$ \\
\hline $\mathrm{C}(3)-\mathrm{C}(4)-\mathrm{W}(1)$ & $71.9(11)$ \\
\hline $\mathrm{C}(5)-\mathrm{C}(4)-\mathrm{W}(1)$ & $73.5(11)$ \\
\hline$C(4)-C(5)-C(1)$ & $105(3)$ \\
\hline
\end{tabular}




\begin{tabular}{|c|c|}
\hline $\mathrm{C}(4)-\mathrm{C}(5)-\mathrm{W}(1)$ & $73.6(13)$ \\
\hline $\mathrm{C}(1)-\mathrm{C}(5)-\mathrm{W}(1)$ & $71.2(12)$ \\
\hline$C(10)-C(6)-C(7)$ & $112.2(16)$ \\
\hline $\mathrm{C}(10)-\mathrm{C}(6)-\mathrm{W}(2)$ & 75.7(9) \\
\hline$C(7)-C(6)-W(2)$ & $73.0(8)$ \\
\hline $\mathrm{C}(8)-\mathrm{C}(7)-\mathrm{C}(6)$ & $105.8(16)$ \\
\hline $\mathrm{C}(8)-\mathrm{C}(7)-\mathrm{W}(2)$ & $74.0(9)$ \\
\hline$C(6)-C(7)-W(2)$ & $72.6(9)$ \\
\hline $\mathrm{C}(7)-\mathrm{C}(8)-\mathrm{C}(9)$ & $106.5(14)$ \\
\hline $\mathrm{C}(7)-\mathrm{C}(8)-\mathrm{W}(2)$ & $71.9(9)$ \\
\hline $\mathrm{C}(9)-\mathrm{C}(8)-\mathrm{W}(2)$ & 72.3(9) \\
\hline $\mathrm{C}(10)-\mathrm{C}(9)-\mathrm{C}(8)$ & $109.7(15)$ \\
\hline $\mathrm{C}(10)-\mathrm{C}(9)-\mathrm{W}(2)$ & 74.4(9) \\
\hline $\mathrm{C}(8)-\mathrm{C}(9)-\mathrm{W}(2)$ & $73.2(9)$ \\
\hline $\mathrm{C}(6)-\mathrm{C}(10)-\mathrm{C}(9)$ & $105.7(15)$ \\
\hline$C(6)-C(10)-W(2)$ & $71.6(8)$ \\
\hline$C(9)-C(10)-W(2)$ & $72.0(8)$ \\
\hline $\mathrm{C}(12)-\mathrm{C}(11)-\mathrm{P}(2)$ & $118.5(12)$ \\
\hline $\mathrm{C}(12)-\mathrm{C}(11)-\mathrm{W}(1)$ & $129.8(12)$ \\
\hline $\mathrm{P}(2)-\mathrm{C}(11)-\mathrm{W}(1)$ & $111.7(7)$ \\
\hline $\mathrm{C}(14)-\mathrm{C}(13)-\mathrm{P}(1)$ & $115.9(11)$ \\
\hline $\mathrm{C}(14)-\mathrm{C}(13)-\mathrm{W}(2)$ & $130.2(11)$ \\
\hline $\mathrm{P}(1)-\mathrm{C}(13)-\mathrm{W}(2)$ & $113.4(7)$ \\
\hline $\mathrm{C}(22)-\mathrm{C}(21)-\mathrm{C}(26)$ & $119.7(16)$ \\
\hline $\mathrm{C}(22)-\mathrm{C}(21)-\mathrm{P}(1)$ & $119.5(12)$ \\
\hline $\mathrm{C}(26)-\mathrm{C}(21)-\mathrm{P}(1)$ & $120.6(15)$ \\
\hline $\mathrm{C}(21)-\mathrm{C}(22)-\mathrm{C}(23)$ & $121.5(17)$ \\
\hline $\mathrm{C}(24)-\mathrm{C}(23)-\mathrm{C}(22)$ & $120(2)$ \\
\hline $\mathrm{C}(25)-\mathrm{C}(24)-\mathrm{C}(23)$ & $119(2)$ \\
\hline $\mathrm{C}(24)-\mathrm{C}(25)-\mathrm{C}(26)$ & $123.0(19)$ \\
\hline $\mathrm{C}(21)-\mathrm{C}(26)-\mathrm{C}(25)$ & $117.2(18)$ \\
\hline $\mathrm{C}(32)-\mathrm{C}(31)-\mathrm{C}(36)$ & $116.5(16)$ \\
\hline $\mathrm{C}(32)-\mathrm{C}(31)-\mathrm{P}(1)$ & $117.8(12)$ \\
\hline $\mathrm{C}(36)-\mathrm{C}(31)-\mathrm{P}(1)$ & $125.3(14)$ \\
\hline $\mathrm{C}(31)-\mathrm{C}(32)-\mathrm{C}(33)$ & $121.5(17)$ \\
\hline $\mathrm{C}(34)-\mathrm{C}(33)-\mathrm{C}(32)$ & $121(2)$ \\
\hline $\mathrm{C}(35)-\mathrm{C}(34)-\mathrm{C}(33)$ & $117.4(19)$ \\
\hline $\mathrm{C}(34)-\mathrm{C}(35)-\mathrm{C}(36)$ & 121.1(18) \\
\hline $\mathrm{C}(35)-\mathrm{C}(36)-\mathrm{C}(31)$ & $122.0(18)$ \\
\hline
\end{tabular}




\begin{tabular}{|c|c|}
\hline$C(42)-C(41)-C(46)$ & $115.8(14)$ \\
\hline $\mathrm{C}(42)-\mathrm{C}(41)-\mathrm{P}(2)$ & $120.3(12)$ \\
\hline $\mathrm{C}(46)-\mathrm{C}(41)-\mathrm{P}(2)$ & $123.9(11)$ \\
\hline $\mathrm{C}(41)-\mathrm{C}(42)-\mathrm{C}(43)$ & $122.9(15)$ \\
\hline $\mathrm{C}(44)-\mathrm{C}(43)-\mathrm{C}(42)$ & $121.2(15)$ \\
\hline $\mathrm{C}(43)-\mathrm{C}(44)-\mathrm{C}(45)$ & $118.0(16)$ \\
\hline $\mathrm{C}(44)-\mathrm{C}(45)-\mathrm{C}(46)$ & $119.5(15)$ \\
\hline $\mathrm{C}(41)-\mathrm{C}(46)-\mathrm{C}(45)$ & $122.7(14)$ \\
\hline$C(56)-C(51)-C(52)$ & $117.3(12)$ \\
\hline $\mathrm{C}(56)-\mathrm{C}(51)-\mathrm{P}(2)$ & $119.0(12)$ \\
\hline $\mathrm{C}(52)-\mathrm{C}(51)-\mathrm{P}(2)$ & $122.6(12)$ \\
\hline $\mathrm{C}(51)-\mathrm{C}(52)-\mathrm{C}(53)$ & $120.3(15)$ \\
\hline$C(54)-C(53)-C(52)$ & $121.8(16)$ \\
\hline $\mathrm{C}(53)-\mathrm{C}(54)-\mathrm{C}(55)$ & $118.6(15)$ \\
\hline$C(56)-C(55)-C(54)$ & $120.0(16)$ \\
\hline$C(51)-C(56)-C(55)$ & $121.9(14)$ \\
\hline $\mathrm{N}(1 \mathrm{~B})-\mathrm{W}(1 \mathrm{~B})-\mathrm{O}(12 \mathrm{~B})$ & $118.5(4)$ \\
\hline $\mathrm{N}(1 \mathrm{~B})-\mathrm{W}(1 \mathrm{~B})-\mathrm{C}(11 \mathrm{~B})$ & $84.2(5)$ \\
\hline $\mathrm{O}(12 \mathrm{~B})-\mathrm{W}(1 \mathrm{~B})-\mathrm{C}(11 \mathrm{~B})$ & $76.4(4)$ \\
\hline $\mathrm{N}(1 \mathrm{~B})-\mathrm{W}(1 \mathrm{~B})-\mathrm{C}(3 \mathrm{~B})$ & $138.2(11)$ \\
\hline $\mathrm{O}(12 \mathrm{~B})-\mathrm{W}(1 \mathrm{~B})-\mathrm{C}(3 \mathrm{~B})$ & $99.4(8)$ \\
\hline $\mathrm{C}(11 \mathrm{~B})-\mathrm{W}(1 \mathrm{~B})-\mathrm{C}(3 \mathrm{~B})$ & $123.9(12)$ \\
\hline $\mathrm{N}(1 \mathrm{~B})-\mathrm{W}(1 \mathrm{~B})-\mathrm{C}(5 \mathrm{~B})$ & $89.8(6)$ \\
\hline $\mathrm{O}(12 \mathrm{~B})-\mathrm{W}(1 \mathrm{~B})-\mathrm{C}(5 \mathrm{~B})$ & $151.3(7)$ \\
\hline $\mathrm{C}(11 \mathrm{~B})-\mathrm{W}(1 \mathrm{~B})-\mathrm{C}(5 \mathrm{~B})$ & $103.8(13)$ \\
\hline $\mathrm{C}(3 \mathrm{~B})-\mathrm{W}(1 \mathrm{~B})-\mathrm{C}(5 \mathrm{~B})$ & $56.1(8)$ \\
\hline $\mathrm{N}(1 \mathrm{~B})-\mathrm{W}(1 \mathrm{~B})-\mathrm{C}(1 \mathrm{~B})$ & $110.8(10)$ \\
\hline $\mathrm{O}(12 \mathrm{~B})-\mathrm{W}(1 \mathrm{~B})-\mathrm{C}(1 \mathrm{~B})$ & $122.6(10)$ \\
\hline $\mathrm{C}(11 \mathrm{~B})-\mathrm{W}(1 \mathrm{~B})-\mathrm{C}(1 \mathrm{~B})$ & $81.3(7)$ \\
\hline $\mathrm{C}(3 \mathrm{~B})-\mathrm{W}(1 \mathrm{~B})-\mathrm{C}(1 \mathrm{~B})$ & $53.7(7)$ \\
\hline $\mathrm{C}(5 \mathrm{~B})-\mathrm{W}(1 \mathrm{~B})-\mathrm{C}(1 \mathrm{~B})$ & $32.7(8)$ \\
\hline $\mathrm{N}(1 \mathrm{~B})-\mathrm{W}(1 \mathrm{~B})-\mathrm{C}(4 \mathrm{~B})$ & $107.4(13)$ \\
\hline $\mathrm{O}(12 \mathrm{~B})-\mathrm{W}(1 \mathrm{~B})-\mathrm{C}(4 \mathrm{~B})$ & $127.3(15)$ \\
\hline$C(11 B)-W(1 B)-C(4 B)$ & $135.3(8)$ \\
\hline $\mathrm{C}(3 \mathrm{~B})-\mathrm{W}(1 \mathrm{~B})-\mathrm{C}(4 \mathrm{~B})$ & $30.8(9)$ \\
\hline $\mathrm{C}(5 \mathrm{~B})-\mathrm{W}(1 \mathrm{~B})-\mathrm{C}(4 \mathrm{~B})$ & $35.9(9)$ \\
\hline $\mathrm{C}(1 \mathrm{~B})-\mathrm{W}(1 \mathrm{~B})-\mathrm{C}(4 \mathrm{~B})$ & $54.1(8)$ \\
\hline $\mathrm{N}(1 \mathrm{~B})-\mathrm{W}(1 \mathrm{~B})-\mathrm{C}(2 \mathrm{~B})$ & $143.2(10)$ \\
\hline $\mathrm{O}(12 \mathrm{~B})-\mathrm{W}(1 \mathrm{~B})-\mathrm{C}(2 \mathrm{~B})$ & $95.6(7)$ \\
\hline
\end{tabular}




\begin{tabular}{|c|c|}
\hline $\mathrm{C}(11 \mathrm{~B})-\mathrm{W}(1 \mathrm{~B})-\mathrm{C}(2 \mathrm{~B})$ & $91.5(11)$ \\
\hline $\mathrm{C}(3 \mathrm{~B})-\mathrm{W}(1 \mathrm{~B})-\mathrm{C}(2 \mathrm{~B})$ & $32.5(8)$ \\
\hline $\mathrm{C}(5 \mathrm{~B})-\mathrm{W}(1 \mathrm{~B})-\mathrm{C}(2 \mathrm{~B})$ & $55.8(7)$ \\
\hline $\mathrm{C}(1 \mathrm{~B})-\mathrm{W}(1 \mathrm{~B})-\mathrm{C}(2 \mathrm{~B})$ & $32.8(7)$ \\
\hline $\mathrm{C}(4 \mathrm{~B})-\mathrm{W}(1 \mathrm{~B})-\mathrm{C}(2 \mathrm{~B})$ & $53.3(8)$ \\
\hline N(1B)-W(1B)-P(1B) & $89.2(4)$ \\
\hline $\mathrm{O}(12 \mathrm{~B})-\mathrm{W}(1 \mathrm{~B})-\mathrm{P}(1 \mathrm{~B})$ & $69.9(2)$ \\
\hline $\mathrm{C}(11 \mathrm{~B})-\mathrm{W}(1 \mathrm{~B})-\mathrm{P}(1 \mathrm{~B})$ & $137.2(4)$ \\
\hline $\mathrm{C}(3 \mathrm{~B})-\mathrm{W}(1 \mathrm{~B})-\mathrm{P}(1 \mathrm{~B})$ & $87.8(9)$ \\
\hline $\mathrm{C}(5 \mathrm{~B})-\mathrm{W}(1 \mathrm{~B})-\mathrm{P}(1 \mathrm{~B})$ & $118.4(13)$ \\
\hline $\mathrm{C}(1 \mathrm{~B})-\mathrm{W}(1 \mathrm{~B})-\mathrm{P}(1 \mathrm{~B})$ & $139.6(5)$ \\
\hline $\mathrm{C}(4 \mathrm{~B})-\mathrm{W}(1 \mathrm{~B})-\mathrm{P}(1 \mathrm{~B})$ & $87.0(9)$ \\
\hline $\mathrm{C}(2 \mathrm{~B})-\mathrm{W}(1 \mathrm{~B})-\mathrm{P}(1 \mathrm{~B})$ & $117.0(12)$ \\
\hline $\mathrm{O}(1 \mathrm{~B})-\mathrm{N}(1 \mathrm{~B})-\mathrm{W}(1 \mathrm{~B})$ & $176.8(10)$ \\
\hline $\mathrm{C}(31 \mathrm{~B})-\mathrm{P}(1 \mathrm{~B})-\mathrm{C}(13 \mathrm{~B})$ & $108.4(7)$ \\
\hline $\mathrm{C}(31 \mathrm{~B})-\mathrm{P}(1 \mathrm{~B})-\mathrm{C}(21 \mathrm{~B})$ & $99.5(6)$ \\
\hline $\mathrm{C}(13 \mathrm{~B})-\mathrm{P}(1 \mathrm{~B})-\mathrm{C}(21 \mathrm{~B})$ & $110.4(6)$ \\
\hline $\mathrm{C}(31 \mathrm{~B})-\mathrm{P}(1 \mathrm{~B})-\mathrm{W}(1 \mathrm{~B})$ & $119.3(5)$ \\
\hline $\mathrm{C}(13 \mathrm{~B})-\mathrm{P}(1 \mathrm{~B})-\mathrm{W}(1 \mathrm{~B})$ & $102.2(5)$ \\
\hline $\mathrm{C}(21 \mathrm{~B})-\mathrm{P}(1 \mathrm{~B})-\mathrm{W}(1 \mathrm{~B})$ & $117.0(4)$ \\
\hline $\mathrm{N}(2 \mathrm{~B})-\mathrm{W}(2 \mathrm{~B})-\mathrm{O}(12 \mathrm{~B})$ & $101.8(4)$ \\
\hline $\mathrm{N}(2 \mathrm{~B})-\mathrm{W}(2 \mathrm{~B})-\mathrm{C}(13 \mathrm{~B})$ & $93.7(5)$ \\
\hline $\mathrm{O}(12 \mathrm{~B})-\mathrm{W}(2 \mathrm{~B})-\mathrm{C}(13 \mathrm{~B})$ & $72.0(4)$ \\
\hline $\mathrm{N}(2 \mathrm{~B})-\mathrm{W}(2 \mathrm{~B})-\mathrm{C}(6 \mathrm{~B})$ & $104.2(5)$ \\
\hline $\mathrm{O}(12 \mathrm{~B})-\mathrm{W}(2 \mathrm{~B})-\mathrm{C}(6 \mathrm{~B})$ & $140.9(4)$ \\
\hline$C(13 B)-W(2 B)-C(6 B)$ & $133.8(5)$ \\
\hline $\mathrm{N}(2 \mathrm{~B})-\mathrm{W}(2 \mathrm{~B})-\mathrm{C}(9 \mathrm{~B})$ & $103.3(5)$ \\
\hline $\mathrm{O}(12 \mathrm{~B})-\mathrm{W}(2 \mathrm{~B})-\mathrm{C}(9 \mathrm{~B})$ & $141.7(4)$ \\
\hline $\mathrm{C}(13 \mathrm{~B})-\mathrm{W}(2 \mathrm{~B})-\mathrm{C}(9 \mathrm{~B})$ & $77.8(5)$ \\
\hline $\mathrm{C}(6 \mathrm{~B})-\mathrm{W}(2 \mathrm{~B})-\mathrm{C}(9 \mathrm{~B})$ & $56.8(5)$ \\
\hline $\mathrm{N}(2 \mathrm{~B})-\mathrm{W}(2 \mathrm{~B})-\mathrm{C}(8 \mathrm{~B})$ & $137.2(6)$ \\
\hline $\mathrm{O}(12 \mathrm{~B})-\mathrm{W}(2 \mathrm{~B})-\mathrm{C}(8 \mathrm{~B})$ & $116.8(4)$ \\
\hline $\mathrm{C}(13 \mathrm{~B})-\mathrm{W}(2 \mathrm{~B})-\mathrm{C}(8 \mathrm{~B})$ & $82.1(5)$ \\
\hline $\mathrm{C}(6 \mathrm{~B})-\mathrm{W}(2 \mathrm{~B})-\mathrm{C}(8 \mathrm{~B})$ & $55.8(5)$ \\
\hline $\mathrm{C}(9 \mathrm{~B})-\mathrm{W}(2 \mathrm{~B})-\mathrm{C}(8 \mathrm{~B})$ & $34.1(5)$ \\
\hline $\mathrm{N}(2 \mathrm{~B})-\mathrm{W}(2 \mathrm{~B})-\mathrm{C}(10 \mathrm{~B})$ & $86.4(5)$ \\
\hline $\mathrm{O}(12 \mathrm{~B})-\mathrm{W}(2 \mathrm{~B})-\mathrm{C}(10 \mathrm{~B})$ & $171.8(4)$ \\
\hline$C(13 B)-W(2 B)-C(10 B)$ & $108.1(6)$ \\
\hline$C(6 B)-W(2 B)-C(10 B)$ & $34.0(4)$ \\
\hline
\end{tabular}




$\begin{array}{lc}\text { C(9B)-W(2B)-C(10B) } & 33.8(4) \\ \text { C(8B)-W(2B)-C(10B) } & 55.6(5) \\ \text { N(2B)-W(2B)-C(7B) } & 139.7(5) \\ \text { O(12B)-W(2B)-C(7B) } & 114.9(4) \\ \text { C(13B)-W(2B)-C(7B) } & 112.4(5) \\ \text { C(6B)-W(2B)-C(7B) } & 35.7(4) \\ \text { C(9B)-W(2B)-C(7B) } & 56.7(5) \\ \text { C(8B)-W(2B)-C(7B) } & 32.3(4) \\ \text { C(10B)-W(2B)-C(7B) } & 57.2(5) \\ \text { N(2B)-W(2B)-P(2B) } & 98.4(4) \\ \text { O(12B)-W(2B)-P(2B) } & 63.5(2) \\ \text { C(13B)-W(2B)-P(2B) } & 135.4(4) \\ \text { C(6B)-W(2B)-P(2B) } & 84.1(4) \\ \text { C(9B)-W(2B)-P(2B) } & 138.8(3) \\ \text { C(8B)-W(2B)-P(2B) } & 114.3(4) \\ \text { C(10B)-W(2B)-P(2B) } & 115.4(4) \\ \text { C(7B)-W(2B)-P(2B) } & 84.3(4) \\ \text { O(2B)-N(2B)-W(2B) } & 167.9(10) \\ \text { C(11B)-P(2B)-C(41B) } & 106.4(7) \\ \text { C(11B)-P(2B)-C(51B) } & 109.7(6) \\ \text { C(41B)-P(2B)-C(51B) } & 101.1(6) \\ \text { C(11B)-P(2B)-W(2B) } & 100.9(5) \\ \text { C(41B)-P(2B)-W(2B) } & 114.9(5) \\ \text { C(51B)-P(2B)-W(2B) } & 123.0(5) \\ \text { W(1B)-O(12B)-W(2B) } & 117.8(3) \\ \text { C(12B)-C(11B)-P(2B) } & 117.3(11) \\ \text { C(12B)-C(11B)-W(1B) } & 129.4(10) \\ \text { P(2B)-C(11B)-W(1B) } & 113.1(6) \\ \text { C(14B)-C(13B)-P(1B) } & 116.9(11) \\ \text { C(14B)-C(2B)-W(1B) } & 127.3(10) \\ \text { P(1B)-C(13B)-W(2B) } & 115.3(8) \\ \text { C(5B)-C(1B)-C(2B) } & 113(2) \\ \text { C(5B)-C(1B)-W(1B) } & 73.1(14) \\ \text { C(2B)-C(1B)-W(1B) } & 76.9(13) \\ \text { C(3B)-C(2B)-C(1B) } & 103(2) \\ \text { C(3B)-C(2B)-W(1B) } & 69.5(15) \\ & 70.3(11) \\ \text { C } 3.4(17)\end{array}$




$\begin{array}{lc}\text { C(2B)-C(3B)-W(1B) } & 78.0(14) \\ \text { C(3B)-C(4B)-C(5B) } & 107(2) \\ \text { C(3B)-C(4B)-W(1B) } & 70.8(13) \\ \text { C(5B)-C(4B)-W(1B) } & 69.1(12) \\ \text { C(1B)-C(5B)-C(4B) } & 102(2) \\ \text { C(1B)-C(5B)-W(1B) } & 74.2(14) \\ \text { C(4B)-C(5B)-W(1B) } & 75.0(12) \\ \text { C(10B)-C(6B)-C(7B) } & 108.0(13) \\ \text { C(10B)-C(6B)-W(2B) } & 75.0(8) \\ \text { C(7B)-C(6B)-W(2B) } & 74.5(7) \\ \text { C(8B)-C(7B)-C(6B) } & 104.9(13) \\ \text { C(8B)-C(7B)-W(2B) } & 72.9(7) \\ \text { C(6B)-C(7B)-W(2B) } & 69.8(6) \\ \text { C(7B)-C(8B)-C(9B) } & 112.3(14) \\ \text { C(7B)-C(8B)-W(2B) } & 74.8(8) \\ \text { C(9B)-C(8B)-W(2B) } & 71.9(8) \\ \text { C(10B)-C(9B)-C(8B) } & 106.8(14) \\ \text { C(10B)-C(9B)-W(2B) } & 74.5(9) \\ \text { C(8B)-C(9B)-W(2B) } & 73.9(9) \\ \text { C(9B)-C(10B)-C(6B) } & 107.8(14) \\ \text { C(9B)-C(10B)-W(2B) } & 71.6(9) \\ \text { C(6B)-C(10B)-W(2B) } & 71.0(8) \\ \text { C(26B)-C(21B)-C(22B) } & 117.8(12) \\ \text { C(26B)-C(21B)-P(1B) } & 122.4(11) \\ \text { C(22B)-C(21B)-P(1B) } & 119.8(9) \\ \text { C(23B)-C(22B)-C(21B) } & 121.2(13) \\ \text { C(24B)-C(23B)-C(22B) } & 119.3(14) \\ \text { C(25B)-C(24B)-C(23B) } & 120.7(14) \\ \text { C(24B)-C(25B)-C(26B) } & 120.2(16) \\ \text { C(21B)-C(26B)-C(25B) } & 120.8(14) \\ \text { C(32B)-C(31B)-C(36B) } & 116.7(14) \\ \text { C(32B)-C(31B)-P(1B) } & 121.9(13) \\ \text { C(36B)-C(31B)-P(1B) } & 121.3(13) \\ \text { C(31B)-C(36B)-C(35B) } & 120.5(18) \\ \text { C(34B)-C(35B)-C(36B) } & 121(2) \\ & 121(2) \\ \text { C(35)-C(34B)-C(33B) } & 118.5(18) \\ & 122.5(18) \\ & 117.8(13) \\ & \end{array}$




$\begin{array}{ll}\mathrm{C}(42 \mathrm{~B})-\mathrm{C}(41 \mathrm{~B})-\mathrm{P}(2 \mathrm{~B}) & 121.5(13) \\ \mathrm{C}(46 \mathrm{~B})-\mathrm{C}(41 \mathrm{~B})-\mathrm{P}(2 \mathrm{~B}) & 120.7(11) \\ \mathrm{C}(43 \mathrm{~B})-\mathrm{C}(42 \mathrm{~B})-\mathrm{C}(41 \mathrm{~B}) & 120.2(16) \\ \mathrm{C}(42 \mathrm{~B})-\mathrm{C}(43 \mathrm{~B})-\mathrm{C}(44 \mathrm{~B}) & 121.7(16) \\ \mathrm{C}(45 \mathrm{~B})-\mathrm{C}(44 \mathrm{~B})-\mathrm{C}(43 \mathrm{~B}) & 119.9(17) \\ \mathrm{C}(44 \mathrm{~B})-\mathrm{C}(45 \mathrm{~B})-\mathrm{C}(46 \mathrm{~B}) & 120.5(18) \\ \mathrm{C}(41 \mathrm{~B})-\mathrm{C}(46 \mathrm{~B})-\mathrm{C}(45 \mathrm{~B}) & 119.9(15) \\ \mathrm{C}(56 \mathrm{~B})-\mathrm{C}(51 \mathrm{~B})-\mathrm{C}(52 \mathrm{~B}) & 116.0(10) \\ \mathrm{C}(56 \mathrm{~B})-\mathrm{C}(51 \mathrm{~B})-\mathrm{P}(2 \mathrm{~B}) & 121.4(9) \\ \mathrm{C}(52 \mathrm{~B})-\mathrm{C}(51 \mathrm{~B})-\mathrm{P}(2 \mathrm{~B}) & 121.9(9) \\ \mathrm{C}(53 \mathrm{~B})-\mathrm{C}(52 \mathrm{~B})-\mathrm{C}(51 \mathrm{~B}) & 122.1(12) \\ \mathrm{C}(54 \mathrm{~B})-\mathrm{C}(53 \mathrm{~B})-\mathrm{C}(52 \mathrm{~B}) & 120.5(13) \\ \mathrm{C}(53 \mathrm{~B})-\mathrm{C}(54 \mathrm{~B})-\mathrm{C}(55 \mathrm{~B}) & 120.3(12) \\ \mathrm{C}(54 \mathrm{~B})-\mathrm{C}(55 \mathrm{~B})-\mathrm{C}(56 \mathrm{~B}) & 118.3(12) \\ \mathrm{C}(51 \mathrm{~B})-\mathrm{C}(56 \mathrm{~B})-\mathrm{C}(55 \mathrm{~B}) & 122.7(11) \\ \mathrm{Cl}(2)-\mathrm{C}(15)-\mathrm{Cl}(1) & 120.5(16) \\ \mathrm{Cl}(4)-\mathrm{C}(16)-\mathrm{Cl}(3) & 89(2) \\ \mathrm{C}(16) \# 1-\mathrm{Cl}(3)-\mathrm{C}(16) & 84(3) \\ \mathrm{C}(16) \# 1-\mathrm{Cl}(3)-\mathrm{Cl}(4) & 41.9(13) \\ \mathrm{C}(16)-\mathrm{Cl}(3)-\mathrm{Cl}(4) & 41.9(13) \\ \mathrm{C}(16) \# 1-\mathrm{Cl}(4)-\mathrm{C}(16) & 98(4) \\ \mathrm{C}(16) \# 1-\mathrm{Cl}(4)-\mathrm{Cl}(3) & 49(2) \\ \mathrm{C}(16)-\mathrm{Cl}(4)-\mathrm{Cl}(3) & 49(2)\end{array}$

Symmetry transformations used to generate equivalent atoms:

$\# 1-\mathrm{x}, \mathrm{y},-\mathrm{z}+1 / 2$ 
Table 4. Anisotropic displacement parameters $\left(\AA^{2} \times 10^{3}\right)$ for 4 a. The anisotropic displacement factor exponent takes the form: $-2 \pi^{2}\left[h^{2} a^{* 2} U^{11}+\ldots+2 h k a^{*} b^{*} U^{12}\right]$

\begin{tabular}{|c|c|c|c|c|c|c|}
\hline & $\mathrm{U}^{11}$ & $\mathrm{U}^{22}$ & $\mathrm{U}^{33}$ & $\mathrm{U}^{23}$ & $\mathrm{U}^{13}$ & $\mathrm{U}^{12}$ \\
\hline $\mathrm{W}(1)$ & $64(1)$ & $65(1)$ & $53(1)$ & $7(1)$ & $14(1)$ & $20(1)$ \\
\hline $\mathrm{O}(1)$ & $90(8)$ & 103(10) & $61(6)$ & $5(7)$ & $19(5)$ & $7(8)$ \\
\hline $\mathrm{N}(1)$ & $72(9)$ & $89(13)$ & $62(7)$ & $40(7)$ & $23(6)$ & $-31(8)$ \\
\hline $\mathrm{P}(1)$ & $65(3)$ & $59(3)$ & $53(2)$ & $6(2)$ & $3(2)$ & $1(2)$ \\
\hline $\mathrm{W}(2)$ & $51(1)$ & $52(1)$ & $51(1)$ & $-2(1)$ & $10(1)$ & $2(1)$ \\
\hline $\mathrm{O}(2)$ & $73(8)$ & $66(10)$ & $126(9)$ & $-22(7)$ & $11(6)$ & $8(7)$ \\
\hline $\mathrm{N}(2)$ & $67(9)$ & $39(10)$ & $90(9)$ & $-17(7)$ & $3(7)$ & $22(7)$ \\
\hline $\mathrm{P}(2)$ & $49(2)$ & $48(3)$ & $60(2)$ & $12(2)$ & $4(2)$ & $8(2)$ \\
\hline $\mathrm{O}(12)$ & $48(5)$ & $64(7)$ & $56(5)$ & $1(5)$ & $6(4)$ & $9(5)$ \\
\hline $\mathrm{C}(1)$ & $100(18)$ & $190(30)$ & $62(13)$ & $9(16)$ & 17(12) & 18(19) \\
\hline$C(2)$ & $96(19)$ & $160(30)$ & 108(19) & $50(20)$ & $58(16)$ & $100(20)$ \\
\hline$C(3)$ & $120(20)$ & $120(30)$ & $97(17)$ & $14(16)$ & $41(15)$ & $36(18)$ \\
\hline$C(4)$ & $110(20)$ & $240(40)$ & $44(11)$ & $0(20)$ & $49(12)$ & $80(30)$ \\
\hline$C(5)$ & $94(18)$ & $110(20)$ & $97(18)$ & $17(16)$ & $58(15)$ & $15(15)$ \\
\hline$C(6)$ & $89(14)$ & $79(14)$ & $50(9)$ & $3(9)$ & $27(9)$ & $-16(11)$ \\
\hline$C(7)$ & $89(13)$ & $79(16)$ & $69(11)$ & $20(10)$ & $22(11)$ & $10(11)$ \\
\hline $\mathrm{C}(8)$ & $126(16)$ & 41(13) & $74(11)$ & $-15(8)$ & 53(13) & $-8(11)$ \\
\hline $\mathrm{C}(9)$ & $72(11)$ & $128(18)$ & $37(8)$ & $-4(9)$ & $19(8)$ & $-24(12)$ \\
\hline$C(10)$ & $75(13)$ & $72(15)$ & $72(11)$ & $-8(9)$ & $28(9)$ & $8(10)$ \\
\hline $\mathrm{C}(11)$ & $52(9)$ & $36(11)$ & $90(10)$ & $16(8)$ & $22(8)$ & $19(8)$ \\
\hline $\mathrm{C}(12)$ & $118(15)$ & $68(15)$ & $73(10)$ & $-6(9)$ & $29(10)$ & $15(12)$ \\
\hline$C(13)$ & $59(10)$ & $57(13)$ & $70(9)$ & $-11(8)$ & $17(8)$ & $18(9)$ \\
\hline$C(14)$ & $106(15)$ & $87(14)$ & $63(9)$ & $-9(9)$ & $38(9)$ & $10(12)$ \\
\hline$C(21)$ & $67(10)$ & $64(14)$ & $66(11)$ & 11(9) & $9(8)$ & $1(10)$ \\
\hline$C(22)$ & $64(11)$ & $73(16)$ & $77(11)$ & $28(11)$ & $8(9)$ & $16(10)$ \\
\hline$C(23)$ & $104(15)$ & $72(16)$ & $114(15)$ & $46(13)$ & $-30(12)$ & $-11(12)$ \\
\hline $\mathrm{C}(24)$ & 112(17) & $90(20)$ & $140(20)$ & $63(17)$ & $-22(15)$ & $-7(15)$ \\
\hline$C(25)$ & $63(12)$ & $120(20)$ & $94(13)$ & $59(14)$ & $35(10)$ & $15(13)$ \\
\hline$C(26)$ & $52(10)$ & $94(16)$ & $91(12)$ & $22(10)$ & $17(8)$ & 11(10) \\
\hline$C(31)$ & $76(11)$ & $41(12)$ & $59(9)$ & $-4(8)$ & $1(8)$ & $28(9)$ \\
\hline$C(32)$ & $88(13)$ & $75(16)$ & $60(10)$ & $-26(10)$ & $2(9)$ & $21(12)$ \\
\hline$C(33)$ & $106(15)$ & $77(16)$ & 107(14) & $-12(13)$ & 11(13) & $-2(14)$ \\
\hline$C(34)$ & $99(16)$ & $100(20)$ & $74(12)$ & $-27(11)$ & $22(11)$ & $-4(14)$ \\
\hline$C(35)$ & $72(12)$ & $140(20)$ & $40(9)$ & $-9(11)$ & $20(8)$ & $-10(14)$ \\
\hline
\end{tabular}




\begin{tabular}{|c|c|c|c|c|c|c|}
\hline$C(36)$ & $67(11)$ & $105(17)$ & $60(9)$ & $11(10)$ & $-4(9)$ & $20(11)$ \\
\hline $\mathrm{C}(41)$ & $41(10)$ & $59(12)$ & $59(8)$ & $13(8)$ & $-9(7)$ & $5(8)$ \\
\hline $\mathrm{C}(42)$ & $40(10)$ & $66(13)$ & $96(11)$ & 19(9) & $5(8)$ & $13(9)$ \\
\hline $\mathrm{C}(43)$ & $48(10)$ & 104(18) & $120(13)$ & $22(13)$ & $23(10)$ & $7(12)$ \\
\hline $\mathrm{C}(44)$ & $85(14)$ & $59(15)$ & $90(11)$ & 14(9) & $22(10)$ & $-18(11)$ \\
\hline $\mathrm{C}(45)$ & 71(13) & $66(15)$ & $80(11)$ & 12(9) & $17(9)$ & $-4(10)$ \\
\hline $\mathrm{C}(46)$ & $55(10)$ & $76(14)$ & $67(9)$ & $9(9)$ & $16(8)$ & $3(11)$ \\
\hline $\mathrm{C}(51)$ & $53(9)$ & $46(12)$ & $52(8)$ & $-3(9)$ & $-7(7)$ & $5(8)$ \\
\hline $\mathrm{C}(52)$ & $77(11)$ & $56(13)$ & $57(10)$ & $-5(8)$ & $-9(8)$ & $-6(9)$ \\
\hline $\mathrm{C}(53)$ & $86(13)$ & $78(16)$ & $66(12)$ & $11(10)$ & $-9(9)$ & $-1(11)$ \\
\hline $\mathrm{C}(54)$ & $58(10)$ & $95(18)$ & $48(9)$ & $-8(10)$ & $3(7)$ & $1(10)$ \\
\hline $\mathrm{C}(55)$ & $62(11)$ & $64(14)$ & $99(14)$ & $-11(11)$ & $24(9)$ & $21(9)$ \\
\hline $\mathrm{C}(56)$ & $61(10)$ & $63(14)$ & $57(10)$ & $10(8)$ & $17(8)$ & $14(9)$ \\
\hline $\mathrm{W}(1 \mathrm{~B})$ & $40(1)$ & $49(1)$ & $49(1)$ & $3(1)$ & 11(1) & $8(1)$ \\
\hline $\mathrm{N}(1 \mathrm{~B})$ & $30(6)$ & 73(9) & $41(6)$ & $14(6)$ & $-2(5)$ & $6(6)$ \\
\hline $\mathrm{O}(1 \mathrm{~B})$ & $60(7)$ & $105(10)$ & $66(6)$ & $7(6)$ & $-4(5)$ & $0(6)$ \\
\hline $\mathrm{P}(1 \mathrm{~B})$ & $40(2)$ & $48(3)$ & $51(2)$ & $0(2)$ & $13(2)$ & $0(2)$ \\
\hline $\mathrm{W}(2 \mathrm{~B})$ & $39(1)$ & $42(1)$ & $47(1)$ & $1(1)$ & 11(1) & $2(1)$ \\
\hline $\mathrm{N}(2 \mathrm{~B})$ & $49(7)$ & $36(8)$ & $52(6)$ & $-1(5)$ & $5(5)$ & $5(6)$ \\
\hline $\mathrm{O}(2 \mathrm{~B})$ & $55(7)$ & 77(9) & $98(7)$ & $18(6)$ & $-11(6)$ & $16(6)$ \\
\hline $\mathrm{P}(2 \mathrm{~B})$ & $39(2)$ & 41(3) & $47(2)$ & $4(2)$ & 11(2) & $-2(2)$ \\
\hline $\mathrm{O}(12 \mathrm{~B})$ & $35(5)$ & $57(7)$ & $41(4)$ & $6(4)$ & $-1(4)$ & $-9(5)$ \\
\hline$C(11 B)$ & $81(11)$ & $56(11)$ & $29(7)$ & $14(7)$ & $-10(6)$ & $32(9)$ \\
\hline$C(12 B)$ & $32(9)$ & 103(16) & 101(12) & $2(11)$ & $15(8)$ & $25(9)$ \\
\hline$C(13 B)$ & $55(10)$ & $41(11)$ & 66(9) & $-16(7)$ & $-23(7)$ & $13(8)$ \\
\hline$C(14 B)$ & $24(8)$ & $89(14)$ & $96(11)$ & $-4(10)$ & $17(7)$ & $28(9)$ \\
\hline $\mathrm{C}(1 \mathrm{~B})$ & $150(20)$ & $33(14)$ & 111(18) & $4(12)$ & $57(15)$ & $10(14)$ \\
\hline $\mathrm{C}(2 \mathrm{~B})$ & $77(17)$ & $120(30)$ & $180(30)$ & $-100(20)$ & $-37(19)$ & $34(16)$ \\
\hline $\mathrm{C}(3 \mathrm{~B})$ & $140(30)$ & $120(30)$ & $128(17)$ & $53(17)$ & $50(20)$ & $90(20)$ \\
\hline $\mathrm{C}(4 \mathrm{~B})$ & $52(14)$ & $80(20)$ & $390(50)$ & $-40(30)$ & $160(30)$ & $26(15)$ \\
\hline $\mathrm{C}(5 \mathrm{~B})$ & $150(30)$ & $110(30)$ & $140(20)$ & $-83(19)$ & $-60(20)$ & $80(20)$ \\
\hline $\mathrm{C}(6 \mathrm{~B})$ & $56(10)$ & $43(11)$ & 68(9) & $-3(8)$ & $31(8)$ & $18(8)$ \\
\hline $\mathrm{C}(7 \mathrm{~B})$ & $41(9)$ & $60(12)$ & $49(8)$ & $5(7)$ & 11(7) & $-1(8)$ \\
\hline $\mathrm{C}(8 \mathrm{~B})$ & $52(10)$ & $65(13)$ & 61(9) & $-21(8)$ & $14(8)$ & $-17(10)$ \\
\hline $\mathrm{C}(9 \mathrm{~B})$ & $63(11)$ & $83(16)$ & $58(9)$ & $3(8)$ & $27(8)$ & $-3(10)$ \\
\hline $\mathrm{C}(10 \mathrm{~B})$ & $76(12)$ & $45(12)$ & $67(9)$ & $-9(8)$ & $10(8)$ & $15(9)$ \\
\hline$C(21 B)$ & $35(8)$ & $40(10)$ & 66(9) & $6(7)$ & $30(7)$ & $-4(7)$ \\
\hline$C(22 B)$ & $55(9)$ & $57(11)$ & $60(9)$ & $7(8)$ & $6(8)$ & $0(9)$ \\
\hline$C(23 B)$ & $91(14)$ & $82(14)$ & $47(9)$ & $-2(8)$ & $5(9)$ & $4(11)$ \\
\hline
\end{tabular}




\begin{tabular}{|c|c|c|c|c|c|c|}
\hline$C(24 B)$ & $96(14)$ & $114(17)$ & $55(10)$ & $-7(10)$ & $23(10)$ & $-20(13)$ \\
\hline$C(25 B)$ & $79(13)$ & $150(20)$ & $78(12)$ & $-20(12)$ & $30(11)$ & $-14(13)$ \\
\hline$C(26 B)$ & $67(12)$ & $96(16)$ & $64(10)$ & $-11(9)$ & $10(8)$ & $-17(10)$ \\
\hline$C(31 B)$ & $31(8)$ & $60(13)$ & $55(8)$ & $-13(8)$ & $14(7)$ & $-10(8)$ \\
\hline $\mathrm{C}(36 \mathrm{~B})$ & $84(14)$ & $58(16)$ & $139(16)$ & $-12(12)$ & $-6(12)$ & $11(11)$ \\
\hline$C(35 B)$ & $65(13)$ & $40(15)$ & $230(20)$ & $-48(14)$ & $-14(15)$ & $3(11)$ \\
\hline$C(34 B)$ & $42(11)$ & $140(20)$ & $106(14)$ & $-40(15)$ & $-11(11)$ & $-7(14)$ \\
\hline$C(33 B)$ & $57(13)$ & $170(20)$ & $79(12)$ & $27(14)$ & 2(9) & $-40(14)$ \\
\hline $\mathrm{C}(32 \mathrm{~B})$ & $35(9)$ & $113(17)$ & $85(11)$ & $9(10)$ & 4(9) & $-32(10)$ \\
\hline$C(41 B)$ & $44(8)$ & $54(12)$ & $40(7)$ & $-9(7)$ & $9(6)$ & $7(8)$ \\
\hline $\mathrm{C}(42 \mathrm{~B})$ & $67(10)$ & $57(13)$ & $66(9)$ & $-12(9)$ & $-5(8)$ & $-10(9)$ \\
\hline$C(43 B)$ & $116(15)$ & $60(15)$ & $64(11)$ & $2(10)$ & $4(10)$ & $-17(12)$ \\
\hline $\mathrm{C}(44 \mathrm{~B})$ & $96(14)$ & $110(20)$ & $46(10)$ & $-23(11)$ & $-2(9)$ & $-7(14)$ \\
\hline $\mathrm{C}(45 \mathrm{~B})$ & $127(16)$ & $42(13)$ & $102(13)$ & $-13(11)$ & $13(12)$ & $-36(12)$ \\
\hline$C(46 B)$ & $64(11)$ & $61(15)$ & $91(12)$ & $-1(10)$ & $16(9)$ & $14(10)$ \\
\hline $\mathrm{C}(51 \mathrm{~B})$ & 44(9) & $33(9)$ & $49(8)$ & 7(7) & $4(6)$ & $-1(7)$ \\
\hline $\mathrm{C}(52 \mathrm{~B})$ & $39(8)$ & $39(10)$ & $68(9)$ & $15(7)$ & $17(7)$ & $-2(7)$ \\
\hline$C(53 B)$ & $58(10)$ & $60(12)$ & $53(8)$ & $16(7)$ & $25(8)$ & $10(9)$ \\
\hline $\mathrm{C}(54 \mathrm{~B})$ & $71(12)$ & $55(11)$ & $75(11)$ & 9(9) & $47(9)$ & $8(9)$ \\
\hline $\mathrm{C}(55 \mathrm{~B})$ & $38(8)$ & $56(13)$ & $96(11)$ & $7(8)$ & $33(9)$ & $3(7)$ \\
\hline $\mathrm{C}(56 \mathrm{~B})$ & 39(9) & $61(13)$ & $48(7)$ & $-6(6)$ & $8(7)$ & $0(7)$ \\
\hline$C(15)$ & $220(30)$ & $480(60)$ & $122(19)$ & $120(30)$ & $60(20)$ & $210(40)$ \\
\hline $\mathrm{Cl}(1)$ & $128(6)$ & $295(12)$ & $243(8)$ & $82(8)$ & $58(6)$ & $47(7)$ \\
\hline $\mathrm{Cl}(2)$ & $224(10)$ & $372(16)$ & $202(8)$ & 63(9) & $23(7)$ & $0(10)$ \\
\hline$C(16)$ & $50(20)$ & $90(40)$ & $370(80)$ & $10(50)$ & $-10(30)$ & $-20(30)$ \\
\hline $\mathrm{Cl}(3)$ & $1290(100)$ & $240(30)$ & $980(70)$ & 0 & $980(80)$ & 0 \\
\hline $\mathrm{Cl}(4)$ & $560(50)$ & $340(40)$ & $560(40)$ & 0 & $300(40)$ & 0 \\
\hline
\end{tabular}


Figure 2. Molecular structure and atom-numbering scheme for complex $4 \mathrm{~b}$ without $\mathrm{H}$ atoms on $\mathrm{Cp}$ rings and phenyl groups. Thermal ellipsoids are shown at the $30 \%$ probability level.

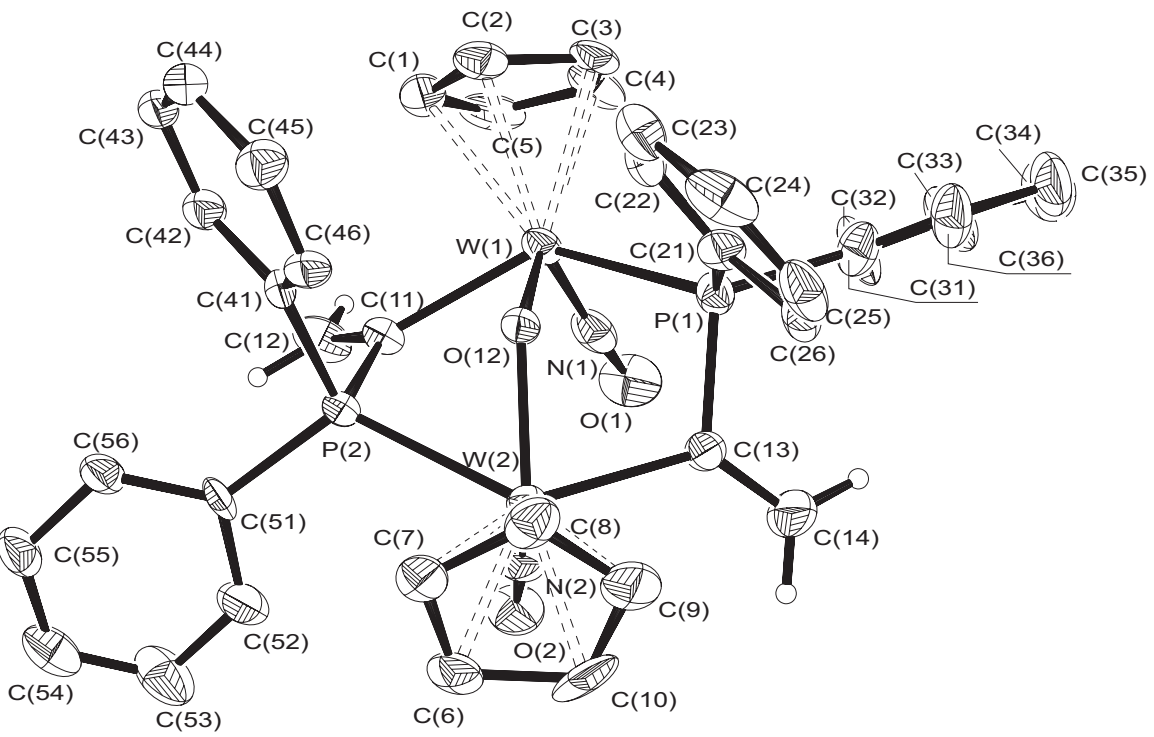


Table 1. Crystal data and structure refinement for $\mathbf{4 b}$.

Identification code

Empirical formula

Formula weight

Temperature

Wavelength

Crystal system

Space group

Unit cell dimensions

Volume

Z

Density (calculated)

Absorption coefficient

$\mathrm{F}(000)$

Crystal size

Theta range for data collection

Index ranges

Reflections collected

Independent reflections

Completeness to theta $=25.96^{\circ}$

Refinement method

Data / restraints / parameters

Goodness-of-fit on $\mathrm{F}^{2}$

Final $\mathrm{R}$ indices $[\mathrm{I}>2 \operatorname{sigma}(\mathrm{I})]$

$\mathrm{R}$ indices (all data)

Largest diff. peak and hole
35

C38 H34 N2 O3 P2 W2

996.31

293(2) K

$0.71073 \AA$

monoclinic

$\mathrm{P} 2{ }_{1} / \mathrm{n}$

$\mathrm{a}=11.2348(11) \AA \quad \alpha=90^{\circ}$.

$\mathrm{b}=23.222(3) \AA \quad \beta=100.037(12)^{\circ}$.

$\mathrm{c}=13.4925(14) \AA \quad \gamma=90^{\circ}$.

3466.3(6) $\AA^{3}$

4

$1.909 \mathrm{Mg} / \mathrm{m}^{3}$

$6.764 \mathrm{~mm}^{-1}$

1912

$0.19 \times 0.12 \times 0.27 \mathrm{~mm}^{3}$

2.18 to $25.96^{\circ}$.

$-12<=\mathrm{h}<=13,-28<=\mathrm{k}<=28,-16<=\mathrm{l}<=16$

24071

$6632[\mathrm{R}(\mathrm{int})=0.0611]$

$97.8 \%$

Full-matrix least-squares on $\mathrm{F}^{2}$

6632 / 0 / 424

1.067

$\mathrm{R} 1=0.0565, \mathrm{wR} 2=0.1479$

$\mathrm{R} 1=0.0859, \mathrm{wR} 2=0.1668$

3.168 and -2.428 e. $\AA^{-3}$ 
Table 2. Atomic coordinates (x 10 $0^{4}$ ) and equivalent isotropic displacement parameters $\left(\AA^{2} \times 10^{3}\right)$ for $\mathbf{4 b .} U(\mathrm{eq})$ is defined as one third of the trace of the orthogonalized $U^{\mathrm{ij}}$ tensor.

\begin{tabular}{|c|c|c|c|c|}
\hline & $\mathrm{x}$ & $\mathrm{y}$ & $\mathrm{z}$ & $\mathrm{U}(\mathrm{eq})$ \\
\hline $\mathrm{W}(1)$ & $1114(1)$ & $1202(1)$ & $1523(1)$ & $38(1)$ \\
\hline $\mathrm{W}(2)$ & $777(1)$ & $1226(1)$ & 4098(1) & $38(1)$ \\
\hline $\mathrm{O}(12)$ & $901(7)$ & $1629(3)$ & $2790(6)$ & $36(2)$ \\
\hline $\mathrm{N}(1)$ & $837(12)$ & $458(5)$ & $1572(8)$ & $55(3)$ \\
\hline $\mathrm{O}(1)$ & 633(13) & $-65(4)$ & 1529(9) & $81(4)$ \\
\hline $\mathrm{N}(2)$ & 1195(12) & $490(4)$ & $4088(7)$ & $50(3)$ \\
\hline $\mathrm{O}(2)$ & $1500(12)$ & $-9(4)$ & 4163(9) & $74(3)$ \\
\hline $\mathrm{P}(1)$ & $-972(3)$ & $1369(1)$ & $1888(2)$ & $41(1)$ \\
\hline $\mathrm{P}(2)$ & 2821(3) & $1445(1)$ & $3734(2)$ & $36(1)$ \\
\hline $\mathrm{C}(11)$ & $2835(12)$ & $1058(5)$ & $2581(8)$ & $39(3)$ \\
\hline $\mathrm{C}(12)$ & $3803(16)$ & $753(6)$ & $2442(11)$ & $64(4)$ \\
\hline$C(13)$ & $-903(12)$ & $986(5)$ & $3080(9)$ & $44(3)$ \\
\hline$C(14)$ & $-1737(17)$ & $615(8)$ & $3200(13)$ & $83(6)$ \\
\hline $\mathrm{C}(21)$ & $-1363(12)$ & $2110(5)$ & $2227(8)$ & $43(3)$ \\
\hline $\mathrm{C}(31)$ & $-2330(14)$ & 1132(6) & $955(11)$ & $54(3)$ \\
\hline$C(41)$ & $3128(10)$ & $2188(5)$ & $3379(8)$ & $36(2)$ \\
\hline$C(51)$ & $4152(11)$ & $1225(5)$ & $4635(8)$ & $41(3)$ \\
\hline$C(22)$ & $-722(12)$ & $2580(5)$ & 1991(10) & $47(3)$ \\
\hline $\mathrm{C}(23)$ & $-964(13)$ & $3123(6)$ & $2357(10)$ & $53(3)$ \\
\hline$C(24)$ & $-1862(17)$ & $3194(7)$ & $2923(10)$ & $64(4)$ \\
\hline$C(25)$ & $-2515(13)$ & $2730(7)$ & $3130(9)$ & $57(4)$ \\
\hline$C(26)$ & $-2276(12)$ & 2181(7) & 2790(9) & $54(3)$ \\
\hline $\mathrm{C}(32)$ & $-2143(17)$ & $639(7)$ & $439(15)$ & $84(6)$ \\
\hline $\mathrm{C}(33)$ & $-3058(19)$ & $433(8)$ & $-283(15)$ & $88(6)$ \\
\hline$C(34)$ & $-4190(20)$ & 711(9) & $-445(16)$ & $95(7)$ \\
\hline$C(35)$ & $-4334(16)$ & $1210(9)$ & $73(13)$ & $83(6)$ \\
\hline$C(36)$ & $-3383(14)$ & $1413(8)$ & $779(12)$ & $67(4)$ \\
\hline $\mathrm{C}(42)$ & $4066(12)$ & $2290(6)$ & 2832(9) & $47(3)$ \\
\hline $\mathrm{C}(43)$ & $4271(12)$ & $2849(6)$ & 2513(9) & $51(3)$ \\
\hline$C(44)$ & $3578(13)$ & $3293(6)$ & $2727(10)$ & $51(3)$ \\
\hline$C(45)$ & 2674(14) & $3200(5)$ & $3269(10)$ & $51(3)$ \\
\hline$C(46)$ & $2464(12)$ & $2649(5)$ & $3601(8)$ & $41(3)$ \\
\hline$C(52)$ & $4063(16)$ & $735(6)$ & $5207(11)$ & $64(4)$ \\
\hline$C(53)$ & $4970(20)$ & $528(7)$ & $5903(15)$ & $88(6)$ \\
\hline
\end{tabular}




\begin{tabular}{lrrrr}
$\mathrm{C}(54)$ & $6044(18)$ & $820(7)$ & $6099(12)$ & $72(5)$ \\
$\mathrm{C}(55)$ & $6174(13)$ & $1321(8)$ & $5600(11)$ & $66(4)$ \\
$\mathrm{C}(56)$ & $5241(12)$ & $1528(7)$ & $4865(9)$ & $52(3)$ \\
$\mathrm{C}(1)$ & $2479(14)$ & $1641(7)$ & $593(10)$ & $56(4)$ \\
$\mathrm{C}(2)$ & $1585(15)$ & $2038(6)$ & $599(9)$ & $55(4)$ \\
$\mathrm{C}(3)$ & $495(14)$ & $1797(6)$ & $108(8)$ & $51(3)$ \\
$\mathrm{C}(4)$ & $773(17)$ & $1258(7)$ & $-224(9)$ & $67(4)$ \\
$\mathrm{C}(5)$ & $2019(18)$ & $1145(7)$ & $92(10)$ & $72(5)$ \\
$\mathrm{C}(6)$ & $1153(15)$ & $1311(6)$ & $5832(9)$ & $56(4)$ \\
$\mathrm{C}(7)$ & $1274(15)$ & $1871(6)$ & $5485(9)$ & $53(3)$ \\
$\mathrm{C}(8)$ & $141(14)$ & $2058(6)$ & $4964(11)$ & $56(4)$ \\
$\mathrm{C}(9)$ & $-681(17)$ & $1629(7)$ & $4991(11)$ & $67(4)$ \\
$\mathrm{C}(10)$ & $-95(18)$ & $1161(7)$ & $5524(11)$ & $76(6)$ \\
\hline
\end{tabular}


Table 3. Bond lengths $[\AA]$ and angles $\left[{ }^{\circ}\right]$ for $\mathbf{4 b}$.

\begin{tabular}{|c|c|}
\hline $\mathrm{W}(1)-\mathrm{N}(1)$ & $1.759(13)$ \\
\hline $\mathrm{W}(1)-\mathrm{O}(12)$ & $2.025(7)$ \\
\hline $\mathrm{W}(1)-\mathrm{C}(11)$ & $2.220(12)$ \\
\hline $\mathrm{W}(1)-\mathrm{C}(4)$ & $2.325(12)$ \\
\hline $\mathrm{W}(1)-\mathrm{C}(5)$ & $2.339(12)$ \\
\hline $\mathrm{W}(1)-\mathrm{C}(3)$ & $2.361(11)$ \\
\hline $\mathrm{W}(1)-\mathrm{C}(1)$ & $2.374(13)$ \\
\hline $\mathrm{W}(1)-\mathrm{C}(2)$ & $2.415(13)$ \\
\hline $\mathrm{W}(1)-\mathrm{P}(1)$ & $2.507(3)$ \\
\hline $\mathrm{W}(2)-\mathrm{N}(2)$ & $1.772(11)$ \\
\hline $\mathrm{W}(2)-\mathrm{O}(12)$ & $2.023(7)$ \\
\hline $\mathrm{W}(2)-\mathrm{C}(13)$ & $2.204(13)$ \\
\hline $\mathrm{W}(2)-\mathrm{C}(10)$ & $2.311(12)$ \\
\hline $\mathrm{W}(2)-\mathrm{C}(6)$ & $2.312(12)$ \\
\hline $\mathrm{W}(2)-\mathrm{C}(7)$ & $2.386(11)$ \\
\hline $\mathrm{W}(2)-\mathrm{C}(9)$ & $2.388(16)$ \\
\hline $\mathrm{W}(2)-\mathrm{C}(8)$ & $2.429(14)$ \\
\hline $\mathrm{W}(2)-\mathrm{P}(2)$ & $2.483(3)$ \\
\hline $\mathrm{N}(1)-\mathrm{O}(1)$ & $1.236(15)$ \\
\hline $\mathrm{N}(2)-\mathrm{O}(2)$ & $1.208(14)$ \\
\hline$P(1)-C(13)$ & $1.828(12)$ \\
\hline$P(1)-C(21)$ & $1.853(12)$ \\
\hline$P(1)-C(31)$ & $1.883(15)$ \\
\hline$P(2)-C(11)$ & $1.800(11)$ \\
\hline$P(2)-C(51)$ & $1.827(11)$ \\
\hline$P(2)-C(41)$ & $1.840(11)$ \\
\hline $\mathrm{C}(11)-\mathrm{C}(12)$ & $1.337(19)$ \\
\hline$C(13)-C(14)$ & $1.304(19)$ \\
\hline $\mathrm{C}(21)-\mathrm{C}(22)$ & $1.375(18)$ \\
\hline$C(21)-C(26)$ & $1.389(18)$ \\
\hline $\mathrm{C}(31)-\mathrm{C}(36)$ & $1.34(2)$ \\
\hline $\mathrm{C}(31)-\mathrm{C}(32)$ & $1.37(2)$ \\
\hline$C(41)-C(46)$ & $1.367(16)$ \\
\hline $\mathrm{C}(41)-\mathrm{C}(42)$ & $1.408(16)$ \\
\hline$C(51)-C(52)$ & $1.389(18)$ \\
\hline$C(51)-C(56)$ & $1.398(19)$ \\
\hline $\mathrm{C}(22)-\mathrm{C}(23)$ & $1.398(18)$ \\
\hline
\end{tabular}




\begin{tabular}{|c|c|}
\hline $\mathrm{C}(23)-\mathrm{C}(24)$ & $1.38(2)$ \\
\hline $\mathrm{C}(24)-\mathrm{C}(25)$ & $1.36(2)$ \\
\hline$C(25)-C(26)$ & $1.40(2)$ \\
\hline $\mathrm{C}(32)-\mathrm{C}(33)$ & $1.37(2)$ \\
\hline$C(33)-C(34)$ & $1.41(3)$ \\
\hline $\mathrm{C}(34)-\mathrm{C}(35)$ & $1.38(3)$ \\
\hline $\mathrm{C}(35)-\mathrm{C}(36)$ & $1.38(2)$ \\
\hline$C(42)-C(43)$ & $1.400(18)$ \\
\hline $\mathrm{C}(43)-\mathrm{C}(44)$ & $1.35(2)$ \\
\hline$C(44)-C(45)$ & $1.37(2)$ \\
\hline $\mathrm{C}(45)-\mathrm{C}(46)$ & $1.389(16)$ \\
\hline$C(52)-C(53)$ & $1.35(2)$ \\
\hline$C(53)-C(54)$ & $1.37(3)$ \\
\hline$C(54)-C(55)$ & $1.37(2)$ \\
\hline $\mathrm{C}(55)-\mathrm{C}(56)$ & $1.397(19)$ \\
\hline $\mathrm{C}(1)-\mathrm{C}(2)$ & $1.37(2)$ \\
\hline $\mathrm{C}(1)-\mathrm{C}(5)$ & $1.39(2)$ \\
\hline $\mathrm{C}(2)-\mathrm{C}(3)$ & $1.40(2)$ \\
\hline$C(3)-C(4)$ & $1.38(2)$ \\
\hline$C(4)-C(5)$ & $1.42(2)$ \\
\hline$C(6)-C(7)$ & $1.397(19)$ \\
\hline$C(6)-C(10)$ & $1.43(2)$ \\
\hline$C(7)-C(8)$ & $1.41(2)$ \\
\hline $\mathrm{C}(8)-\mathrm{C}(9)$ & $1.36(2)$ \\
\hline $\mathrm{C}(9)-\mathrm{C}(10)$ & $1.40(3)$ \\
\hline $\mathrm{N}(1)-\mathrm{W}(1)-\mathrm{O}(12)$ & $113.6(4)$ \\
\hline $\mathrm{N}(1)-\mathrm{W}(1)-\mathrm{C}(11)$ & $88.1(5)$ \\
\hline $\mathrm{O}(12)-\mathrm{W}(1)-\mathrm{C}(11)$ & $75.5(4)$ \\
\hline $\mathrm{N}(1)-\mathrm{W}(1)-\mathrm{C}(4)$ & $95.4(5)$ \\
\hline $\mathrm{O}(12)-\mathrm{W}(1)-\mathrm{C}(4)$ & $143.7(4)$ \\
\hline $\mathrm{C}(11)-\mathrm{W}(1)-\mathrm{C}(4)$ & $129.1(5)$ \\
\hline $\mathrm{N}(1)-\mathrm{W}(1)-\mathrm{C}(5)$ & $94.6(6)$ \\
\hline $\mathrm{O}(12)-\mathrm{W}(1)-\mathrm{C}(5)$ & $149.0(5)$ \\
\hline $\mathrm{C}(11)-\mathrm{W}(1)-\mathrm{C}(5)$ & $93.8(5)$ \\
\hline $\mathrm{C}(4)-\mathrm{W}(1)-\mathrm{C}(5)$ & $35.3(6)$ \\
\hline $\mathrm{N}(1)-\mathrm{W}(1)-\mathrm{C}(3)$ & $125.2(5)$ \\
\hline $\mathrm{O}(12)-\mathrm{W}(1)-\mathrm{C}(3)$ & $109.6(4)$ \\
\hline $\mathrm{C}(11)-\mathrm{W}(1)-\mathrm{C}(3)$ & $135.1(5)$ \\
\hline
\end{tabular}




\begin{tabular}{|c|c|}
\hline $\mathrm{C}(4)-\mathrm{W}(1)-\mathrm{C}(3)$ & $34.3(5)$ \\
\hline $\mathrm{C}(5)-\mathrm{W}(1)-\mathrm{C}(3)$ & $58.3(5)$ \\
\hline $\mathrm{N}(1)-\mathrm{W}(1)-\mathrm{C}(1)$ & $125.2(5)$ \\
\hline $\mathrm{O}(12)-\mathrm{W}(1)-\mathrm{C}(1)$ & $114.8(4)$ \\
\hline $\mathrm{C}(11)-\mathrm{W}(1)-\mathrm{C}(1)$ & $80.4(5)$ \\
\hline $\mathrm{C}(4)-\mathrm{W}(1)-\mathrm{C}(1)$ & $56.4(6)$ \\
\hline $\mathrm{C}(5)-\mathrm{W}(1)-\mathrm{C}(1)$ & $34.3(6)$ \\
\hline $\mathrm{C}(3)-\mathrm{W}(1)-\mathrm{C}(1)$ & $56.5(5)$ \\
\hline $\mathrm{N}(1)-\mathrm{W}(1)-\mathrm{C}(2)$ & $149.9(5)$ \\
\hline $\mathrm{O}(12)-\mathrm{W}(1)-\mathrm{C}(2)$ & $96.4(4)$ \\
\hline $\mathrm{C}(11)-\mathrm{W}(1)-\mathrm{C}(2)$ & $102.0(5)$ \\
\hline $\mathrm{C}(4)-\mathrm{W}(1)-\mathrm{C}(2)$ & $56.2(5)$ \\
\hline $\mathrm{C}(5)-\mathrm{W}(1)-\mathrm{C}(2)$ & $56.9(5)$ \\
\hline $\mathrm{C}(3)-\mathrm{W}(1)-\mathrm{C}(2)$ & $34.1(5)$ \\
\hline $\mathrm{C}(1)-\mathrm{W}(1)-\mathrm{C}(2)$ & $33.1(5)$ \\
\hline $\mathrm{N}(1)-\mathrm{W}(1)-\mathrm{P}(1)$ & $88.1(4)$ \\
\hline $\mathrm{O}(12)-\mathrm{W}(1)-\mathrm{P}(1)$ & $60.5(2)$ \\
\hline $\mathrm{C}(11)-\mathrm{W}(1)-\mathrm{P}(1)$ & $129.6(3)$ \\
\hline $\mathrm{C}(4)-\mathrm{W}(1)-\mathrm{P}(1)$ & $101.3(5)$ \\
\hline $\mathrm{C}(5)-\mathrm{W}(1)-\mathrm{P}(1)$ & $136.6(5)$ \\
\hline $\mathrm{C}(3)-\mathrm{W}(1)-\mathrm{P}(1)$ & $85.1(4)$ \\
\hline $\mathrm{C}(1)-\mathrm{W}(1)-\mathrm{P}(1)$ & $138.6(4)$ \\
\hline $\mathrm{C}(2)-\mathrm{W}(1)-\mathrm{P}(1)$ & $105.9(4)$ \\
\hline $\mathrm{N}(2)-\mathrm{W}(2)-\mathrm{O}(12)$ & $112.4(4)$ \\
\hline $\mathrm{N}(2)-\mathrm{W}(2)-\mathrm{C}(13)$ & $87.2(5)$ \\
\hline $\mathrm{O}(12)-\mathrm{W}(2)-\mathrm{C}(13)$ & $75.8(4)$ \\
\hline $\mathrm{N}(2)-\mathrm{W}(2)-\mathrm{C}(10)$ & $95.4(6)$ \\
\hline $\mathrm{O}(12)-\mathrm{W}(2)-\mathrm{C}(10)$ & $149.3(6)$ \\
\hline $\mathrm{C}(13)-\mathrm{W}(2)-\mathrm{C}(10)$ & $93.5(6)$ \\
\hline $\mathrm{N}(2)-\mathrm{W}(2)-\mathrm{C}(6)$ & $95.1(5)$ \\
\hline $\mathrm{O}(12)-\mathrm{W}(2)-\mathrm{C}(6)$ & $144.6(4)$ \\
\hline $\mathrm{C}(13)-\mathrm{W}(2)-\mathrm{C}(6)$ & $129.6(5)$ \\
\hline $\mathrm{C}(10)-\mathrm{W}(2)-\mathrm{C}(6)$ & $36.1(6)$ \\
\hline $\mathrm{N}(2)-\mathrm{W}(2)-\mathrm{C}(7)$ & $125.8(5)$ \\
\hline $\mathrm{O}(12)-\mathrm{W}(2)-\mathrm{C}(7)$ & $110.6(4)$ \\
\hline $\mathrm{C}(13)-\mathrm{W}(2)-\mathrm{C}(7)$ & $134.8(5)$ \\
\hline $\mathrm{C}(10)-\mathrm{W}(2)-\mathrm{C}(7)$ & $57.5(5)$ \\
\hline$C(6)-W(2)-C(7)$ & $34.5(5)$ \\
\hline $\mathrm{N}(2)-\mathrm{W}(2)-\mathrm{C}(9)$ & $126.0(5)$ \\
\hline
\end{tabular}




\begin{tabular}{|c|c|}
\hline $\mathrm{O}(12)-\mathrm{W}(2)-\mathrm{C}(9)$ & $114.6(5)$ \\
\hline $\mathrm{C}(13)-\mathrm{W}(2)-\mathrm{C}(9)$ & 79.9(5) \\
\hline $\mathrm{C}(10)-\mathrm{W}(2)-\mathrm{C}(9)$ & $34.7(6)$ \\
\hline $\mathrm{C}(6)-\mathrm{W}(2)-\mathrm{C}(9)$ & $58.4(6)$ \\
\hline $\mathrm{C}(7)-\mathrm{W}(2)-\mathrm{C}(9)$ & $56.3(6)$ \\
\hline $\mathrm{N}(2)-\mathrm{W}(2)-\mathrm{C}(8)$ & $150.5(5)$ \\
\hline $\mathrm{O}(12)-\mathrm{W}(2)-\mathrm{C}(8)$ & $97.1(4)$ \\
\hline $\mathrm{C}(13)-\mathrm{W}(2)-\mathrm{C}(8)$ & $102.0(5)$ \\
\hline $\mathrm{C}(10)-\mathrm{W}(2)-\mathrm{C}(8)$ & $56.5(5)$ \\
\hline $\mathrm{C}(6)-\mathrm{W}(2)-\mathrm{C}(8)$ & $57.5(5)$ \\
\hline $\mathrm{C}(7)-\mathrm{W}(2)-\mathrm{C}(8)$ & $34.1(5)$ \\
\hline $\mathrm{C}(9)-\mathrm{W}(2)-\mathrm{C}(8)$ & $32.8(5)$ \\
\hline $\mathrm{N}(2)-\mathrm{W}(2)-\mathrm{P}(2)$ & $86.6(4)$ \\
\hline $\mathrm{O}(12)-\mathrm{W}(2)-\mathrm{P}(2)$ & $61.7(2)$ \\
\hline $\mathrm{C}(13)-\mathrm{W}(2)-\mathrm{P}(2)$ & $130.5(3)$ \\
\hline $\mathrm{C}(10)-\mathrm{W}(2)-\mathrm{P}(2)$ & $136.0(5)$ \\
\hline $\mathrm{C}(6)-\mathrm{W}(2)-\mathrm{P}(2)$ & $99.8(4)$ \\
\hline $\mathrm{C}(7)-\mathrm{W}(2)-\mathrm{P}(2)$ & $85.9(4)$ \\
\hline $\mathrm{C}(9)-\mathrm{W}(2)-\mathrm{P}(2)$ & 139.4(4) \\
\hline $\mathrm{C}(8)-\mathrm{W}(2)-\mathrm{P}(2)$ & 107.0(4) \\
\hline $\mathrm{W}(2)-\mathrm{O}(12)-\mathrm{W}(1)$ & $123.1(4)$ \\
\hline $\mathrm{O}(1)-\mathrm{N}(1)-\mathrm{W}(1)$ & $175.2(10)$ \\
\hline $\mathrm{O}(2)-\mathrm{N}(2)-\mathrm{W}(2)$ & $174.8(10)$ \\
\hline $\mathrm{C}(13)-\mathrm{P}(1)-\mathrm{C}(21)$ & $102.1(5)$ \\
\hline $\mathrm{C}(13)-\mathrm{P}(1)-\mathrm{C}(31)$ & $110.5(6)$ \\
\hline $\mathrm{C}(21)-\mathrm{P}(1)-\mathrm{C}(31)$ & $103.8(6)$ \\
\hline $\mathrm{C}(13)-\mathrm{P}(1)-\mathrm{W}(1)$ & $101.5(5)$ \\
\hline $\mathrm{C}(21)-\mathrm{P}(1)-\mathrm{W}(1)$ & $117.5(4)$ \\
\hline $\mathrm{C}(31)-\mathrm{P}(1)-\mathrm{W}(1)$ & $120.0(5)$ \\
\hline $\mathrm{C}(11)-\mathrm{P}(2)-\mathrm{C}(51)$ & $107.8(5)$ \\
\hline $\mathrm{C}(11)-\mathrm{P}(2)-\mathrm{C}(41)$ & $102.3(5)$ \\
\hline $\mathrm{C}(51)-\mathrm{P}(2)-\mathrm{C}(41)$ & $105.5(6)$ \\
\hline $\mathrm{C}(11)-\mathrm{P}(2)-\mathrm{W}(2)$ & $102.5(4)$ \\
\hline $\mathrm{C}(51)-\mathrm{P}(2)-\mathrm{W}(2)$ & 119.3(4) \\
\hline $\mathrm{C}(41)-\mathrm{P}(2)-\mathrm{W}(2)$ & 117.7(4) \\
\hline $\mathrm{C}(12)-\mathrm{C}(11)-\mathrm{P}(2)$ & $121.0(10)$ \\
\hline $\mathrm{C}(12)-\mathrm{C}(11)-\mathrm{W}(1)$ & $128.9(9)$ \\
\hline $\mathrm{P}(2)-\mathrm{C}(11)-\mathrm{W}(1)$ & $110.0(6)$ \\
\hline $\mathrm{C}(14)-\mathrm{C}(13)-\mathrm{P}(1)$ & $120.6(11)$ \\
\hline
\end{tabular}




$\begin{array}{lc}\mathrm{C}(14)-\mathrm{C}(13)-\mathrm{W}(2) & 130.2(11) \\ \mathrm{P}(1)-\mathrm{C}(13)-\mathrm{W}(2) & 109.0(6) \\ \mathrm{C}(22)-\mathrm{C}(21)-\mathrm{C}(26) & 119.9(12) \\ \mathrm{C}(22)-\mathrm{C}(21)-\mathrm{P}(1) & 121.6(9) \\ \mathrm{C}(26)-\mathrm{C}(21)-\mathrm{P}(1) & 118.4(10) \\ \mathrm{C}(36)-\mathrm{C}(31)-\mathrm{C}(32) & 121.7(14) \\ \mathrm{C}(36)-\mathrm{C}(31)-\mathrm{P}(1) & 124.4(11) \\ \mathrm{C}(32)-\mathrm{C}(31)-\mathrm{P}(1) & 113.9(12) \\ \mathrm{C}(46)-\mathrm{C}(41)-\mathrm{C}(42) & 118.1(11) \\ \mathrm{C}(46)-\mathrm{C}(41)-\mathrm{P}(2) & 122.9(9) \\ \mathrm{C}(42)-\mathrm{C}(41)-\mathrm{P}(2) & 119.0(9) \\ \mathrm{C}(52)-\mathrm{C}(51)-\mathrm{C}(56) & 115.7(12) \\ \mathrm{C}(52)-\mathrm{C}(51)-\mathrm{P}(2) & 117.9(10) \\ \mathrm{C}(56)-\mathrm{C}(51)-\mathrm{P}(2) & 126.1(10) \\ \mathrm{C}(21)-\mathrm{C}(22)-\mathrm{C}(23) & 119.5(13) \\ \mathrm{C}(24)-\mathrm{C}(23)-\mathrm{C}(22) & 120.7(14) \\ \mathrm{C}(25)-\mathrm{C}(24)-\mathrm{C}(23) & 119.5(13) \\ \mathrm{C}(24)-\mathrm{C}(25)-\mathrm{C}(26) & 120.9(13) \\ \mathrm{C}(21)-\mathrm{C}(26)-\mathrm{C}(25) & 119.4(14) \\ \mathrm{C}(33)-\mathrm{C}(32)-\mathrm{C}(31) & 119.2(17) \\ \mathrm{C}(32)-\mathrm{C}(33)-\mathrm{C}(34) & 119.6(17) \\ \mathrm{C}(35)-\mathrm{C}(34)-\mathrm{C}(33) & 119.5(16) \\ \mathrm{C}(34)-\mathrm{C}(35)-\mathrm{C}(36) & 119.3(18) \\ \mathrm{C}(31)-\mathrm{C}(36)-\mathrm{C}(35) & 120.7(16) \\ \mathrm{C}(43)-\mathrm{C}(42)-\mathrm{C}(41) & 119.7(12) \\ \mathrm{C}(44)-\mathrm{C}(43)-\mathrm{C}(42) & 120.6(12) \\ \mathrm{C}(43)-\mathrm{C}(44)-\mathrm{C}(45) & 120.1(12) \\ \mathrm{C}(44)-\mathrm{C}(45)-\mathrm{C}(46) & 120.2(13) \\ \mathrm{C}(41)-\mathrm{C}(46)-\mathrm{C}(45) & 121.4(12) \\ \mathrm{C}(53)-\mathrm{C}(52)-\mathrm{C}(51) & 124.2(17) \\ \mathrm{C}(52)-\mathrm{C}(53)-\mathrm{C}(54) & 119.4(16) \\ \mathrm{C}(55)-\mathrm{C}(54)-\mathrm{C}(53) & 119.5(15) \\ \mathrm{C}(54)-\mathrm{C}(55)-\mathrm{C}(56) & 120.9(16) \\ \mathrm{C}(55)-\mathrm{C}(56)-\mathrm{C}(51) & 120.2(14) \\ \mathrm{C}(2)-\mathrm{C}(1)-\mathrm{C}(5) & 110.7(15) \\ \mathrm{C}(2)-\mathrm{W}(1) & \end{array}$




$\begin{array}{lc}\mathrm{C}(3)-\mathrm{C}(2)-\mathrm{W}(1) & 70.8(7) \\ \mathrm{C}(4)-\mathrm{C}(3)-\mathrm{C}(2) & 106.7(14) \\ \mathrm{C}(4)-\mathrm{C}(3)-\mathrm{W}(1) & 71.4(7) \\ \mathrm{C}(2)-\mathrm{C}(3)-\mathrm{W}(1) & 75.1(7) \\ \mathrm{C}(3)-\mathrm{C}(4)-\mathrm{C}(5) & 109.8(15) \\ \mathrm{C}(3)-\mathrm{C}(4)-\mathrm{W}(1) & 74.3(7) \\ \mathrm{C}(5)-\mathrm{C}(4)-\mathrm{W}(1) & 72.8(7) \\ \mathrm{C}(1)-\mathrm{C}(5)-\mathrm{C}(4) & 104.8(13) \\ \mathrm{C}(1)-\mathrm{C}(5)-\mathrm{W}(1) & 74.3(7) \\ \mathrm{C}(4)-\mathrm{C}(5)-\mathrm{W}(1) & 71.8(8) \\ \mathrm{C}(7)-\mathrm{C}(6)-\mathrm{C}(10) & 106.0(14) \\ \mathrm{C}(7)-\mathrm{C}(6)-\mathrm{W}(2) & 75.6(7) \\ \mathrm{C}(10)-\mathrm{C}(6)-\mathrm{W}(2) & 71.9(7) \\ \mathrm{C}(6)-\mathrm{C}(7)-\mathrm{C}(8) & 108.7(13) \\ \mathrm{C}(6)-\mathrm{C}(7)-\mathrm{W}(2) & 69.8(7) \\ \mathrm{C}(8)-\mathrm{C}(7)-\mathrm{W}(2) & 74.6(7) \\ \mathrm{C}(9)-\mathrm{C}(8)-\mathrm{C}(7) & 108.6(14) \\ \mathrm{C}(9)-\mathrm{C}(8)-\mathrm{W}(2) & 71.9(9) \\ \mathrm{C}(7)-\mathrm{C}(8)-\mathrm{W}(2) & 71.3(8) \\ \mathrm{C}(8)-\mathrm{C}(9)-\mathrm{C}(10) & 108.6(16) \\ \mathrm{C}(8)-\mathrm{C}(9)-\mathrm{W}(2) & 75.2(9) \\ \mathrm{C}(10)-\mathrm{C}(9)-\mathrm{W}(2) & 69.6(9) \\ \mathrm{C}(9)-\mathrm{C}(10)-\mathrm{C}(6) & 108.0(13) \\ \mathrm{C}(9)-\mathrm{C}(10)-\mathrm{W}(2) & 75.7(8) \\ \mathrm{C}(6)-\mathrm{C}(10)-\mathrm{W}(2) & 72.0(7) \\ & \\ & \end{array}$

Symmetry transformations used to generate equivalent atoms: 
Table 4. Anisotropic displacement parameters $\left(\AA^{2} \mathrm{x} 10^{3}\right)$ for $\mathbf{4 b}$. The anisotropic displacement factor exponent takes the form: $-2 \pi^{2}\left[h^{2} a^{* 2} U^{11}+\ldots+2 h k a^{*} b^{*} U^{12}\right]$

\begin{tabular}{|c|c|c|c|c|c|c|}
\hline & $\mathrm{U}^{11}$ & $\mathrm{U}^{22}$ & $\mathrm{U}^{33}$ & $\mathrm{U}^{23}$ & $\mathrm{U}^{13}$ & $\mathrm{U}^{12}$ \\
\hline $\mathrm{W}(1)$ & $42(1)$ & $48(1)$ & $24(1)$ & $-3(1)$ & $4(1)$ & $4(1)$ \\
\hline $\mathrm{W}(2)$ & $42(1)$ & $47(1)$ & $27(1)$ & $1(1)$ & $6(1)$ & $-11(1)$ \\
\hline $\mathrm{O}(12)$ & $36(5)$ & $42(4)$ & $29(4)$ & $1(3)$ & $5(3)$ & $-1(3)$ \\
\hline $\mathrm{N}(1)$ & $57(8)$ & $77(8)$ & $29(5)$ & $-8(5)$ & $0(5)$ & $11(6)$ \\
\hline $\mathrm{O}(1)$ & $122(11)$ & $45(6)$ & $75(8)$ & $-12(5)$ & $12(7)$ & $-4(6)$ \\
\hline $\mathrm{N}(2)$ & $68(8)$ & $48(6)$ & $32(5)$ & $2(4)$ & $1(5)$ & $-20(5)$ \\
\hline $\mathrm{O}(2)$ & $95(10)$ & $43(5)$ & $81(8)$ & $10(5)$ & $5(7)$ & $-8(5)$ \\
\hline $\mathrm{P}(1)$ & $37(2)$ & $50(2)$ & $35(2)$ & $-5(1)$ & $2(1)$ & $-4(1)$ \\
\hline $\mathrm{P}(2)$ & $38(2)$ & $42(1)$ & $26(1)$ & $2(1)$ & $2(1)$ & $-2(1)$ \\
\hline$C(11)$ & $44(8)$ & $39(6)$ & $32(6)$ & $0(4)$ & $4(5)$ & $7(5)$ \\
\hline $\mathrm{C}(12)$ & $92(13)$ & $61(8)$ & $39(7)$ & $-10(6)$ & $7(7)$ & $21(8)$ \\
\hline $\mathrm{C}(13)$ & $47(8)$ & $53(7)$ & $32(6)$ & $-2(5)$ & $3(5)$ & $-16(6)$ \\
\hline $\mathrm{C}(14)$ & $78(14)$ & 109(13) & $55(10)$ & $10(9)$ & $-4(9)$ & $-56(11)$ \\
\hline$C(21)$ & $48(8)$ & $58(7)$ & $24(5)$ & $-3(5)$ & $12(5)$ & $3(6)$ \\
\hline$C(31)$ & 47(9) & $54(7)$ & $62(9)$ & $-17(6)$ & $13(7)$ & $-16(6)$ \\
\hline $\mathrm{C}(41)$ & $24(7)$ & $50(6)$ & $32(6)$ & $5(5)$ & $-2(5)$ & $-5(5)$ \\
\hline $\mathrm{C}(51)$ & $29(7)$ & $60(7)$ & $27(5)$ & $-4(5)$ & $-12(4)$ & $4(5)$ \\
\hline $\mathrm{C}(22)$ & $31(8)$ & $54(7)$ & $55(8)$ & $0(6)$ & $2(6)$ & $11(5)$ \\
\hline$C(23)$ & 47(9) & $53(7)$ & $55(8)$ & $-1(6)$ & $-5(7)$ & $10(6)$ \\
\hline$C(24)$ & $88(13)$ & $66(9)$ & $35(7)$ & $-7(6)$ & $5(7)$ & $36(9)$ \\
\hline$C(25)$ & $40(9)$ & $102(11)$ & $28(6)$ & $-16(7)$ & $-1(6)$ & $18(7)$ \\
\hline$C(26)$ & $33(8)$ & $95(10)$ & $31(6)$ & $-9(6)$ & $1(5)$ & $-3(7)$ \\
\hline$C(32)$ & $59(12)$ & $62(9)$ & $109(15)$ & $-15(9)$ & $-46(10)$ & $6(8)$ \\
\hline$C(33)$ & $87(16)$ & $77(11)$ & $81(12)$ & $-23(9)$ & $-38(11)$ & $3(10)$ \\
\hline$C(34)$ & $86(17)$ & $98(14)$ & $87(14)$ & $-13(11)$ & $-27(11)$ & $-38(12)$ \\
\hline$C(35)$ & $42(10)$ & $143(17)$ & $58(10)$ & $-10(10)$ & $-9(8)$ & $-9(10)$ \\
\hline$C(36)$ & $42(10)$ & $99(11)$ & $58(9)$ & $-28(8)$ & $3(7)$ & $-14(8)$ \\
\hline $\mathrm{C}(42)$ & $39(8)$ & $64(8)$ & $37(6)$ & $8(5)$ & $8(6)$ & $1(6)$ \\
\hline$C(43)$ & $35(8)$ & $86(9)$ & $34(6)$ & $18(6)$ & $5(5)$ & $-10(6)$ \\
\hline$C(44)$ & $45(9)$ & $58(8)$ & $46(7)$ & $16(6)$ & $0(6)$ & $-11(6)$ \\
\hline$C(45)$ & $57(10)$ & $44(6)$ & $53(8)$ & $-1(6)$ & $11(7)$ & $-3(6)$ \\
\hline$C(46)$ & $46(8)$ & $50(6)$ & $29(6)$ & $6(5)$ & $11(5)$ & $-1(5)$ \\
\hline$C(52)$ & $73(12)$ & $70(9)$ & $42(8)$ & $9(7)$ & $-5(7)$ & $0(8)$ \\
\hline$C(53)$ & $108(18)$ & $53(9)$ & $85(13)$ & $13(8)$ & $-32(11)$ & $10(9)$ \\
\hline
\end{tabular}




\begin{tabular}{lcccccc}
$\mathrm{C}(54)$ & $75(13)$ & $80(10)$ & $53(9)$ & $6(8)$ & $-7(8)$ & $29(9)$ \\
$\mathrm{C}(55)$ & $31(8)$ & $120(13)$ & $45(8)$ & $5(8)$ & $1(6)$ & $6(8)$ \\
$\mathrm{C}(56)$ & $34(8)$ & $90(10)$ & $34(6)$ & $12(6)$ & $9(6)$ & $9(6)$ \\
$\mathrm{C}(1)$ & $52(9)$ & $90(10)$ & $30(6)$ & $1(6)$ & $19(6)$ & $-2(7)$ \\
$\mathrm{C}(2)$ & $71(11)$ & $67(8)$ & $30(6)$ & $7(6)$ & $19(7)$ & $7(7)$ \\
$\mathrm{C}(3)$ & $55(9)$ & $79(9)$ & $20(5)$ & $15(5)$ & $9(6)$ & $15(7)$ \\
$\mathrm{C}(4)$ & $92(13)$ & $85(10)$ & $17(6)$ & $-8(6)$ & $-8(6)$ & $16(9)$ \\
$\mathrm{C}(5)$ & $105(15)$ & $89(11)$ & $32(7)$ & $15(7)$ & $36(8)$ & $40(10)$ \\
$\mathrm{C}(6)$ & $77(11)$ & $65(8)$ & $24(6)$ & $-7(5)$ & $5(6)$ & $-7(7)$ \\
$\mathrm{C}(7)$ & $67(10)$ & $64(8)$ & $26(6)$ & $-15(5)$ & $9(6)$ & $-18(7)$ \\
$\mathrm{C}(8)$ & $62(10)$ & $64(8)$ & $47(8)$ & $-8(6)$ & $24(7)$ & $-8(7)$ \\
$\mathrm{C}(9)$ & $79(12)$ & $93(11)$ & $33(7)$ & $-8(7)$ & $19(7)$ & $-12(9)$ \\
$\mathrm{C}(10)$ & $104(15)$ & $87(11)$ & $52(9)$ & $-15(8)$ & $58(10)$ & $-47(10)$ \\
\hline
\end{tabular}


Figure 3. Molecular structure and atom-numbering scheme for complex $\mathbf{5}$ without $\mathrm{H}$ atoms on $\mathrm{Cp}$ rings and phenyl groups. Thermal ellipsoids are shown at the $30 \%$ probability level.

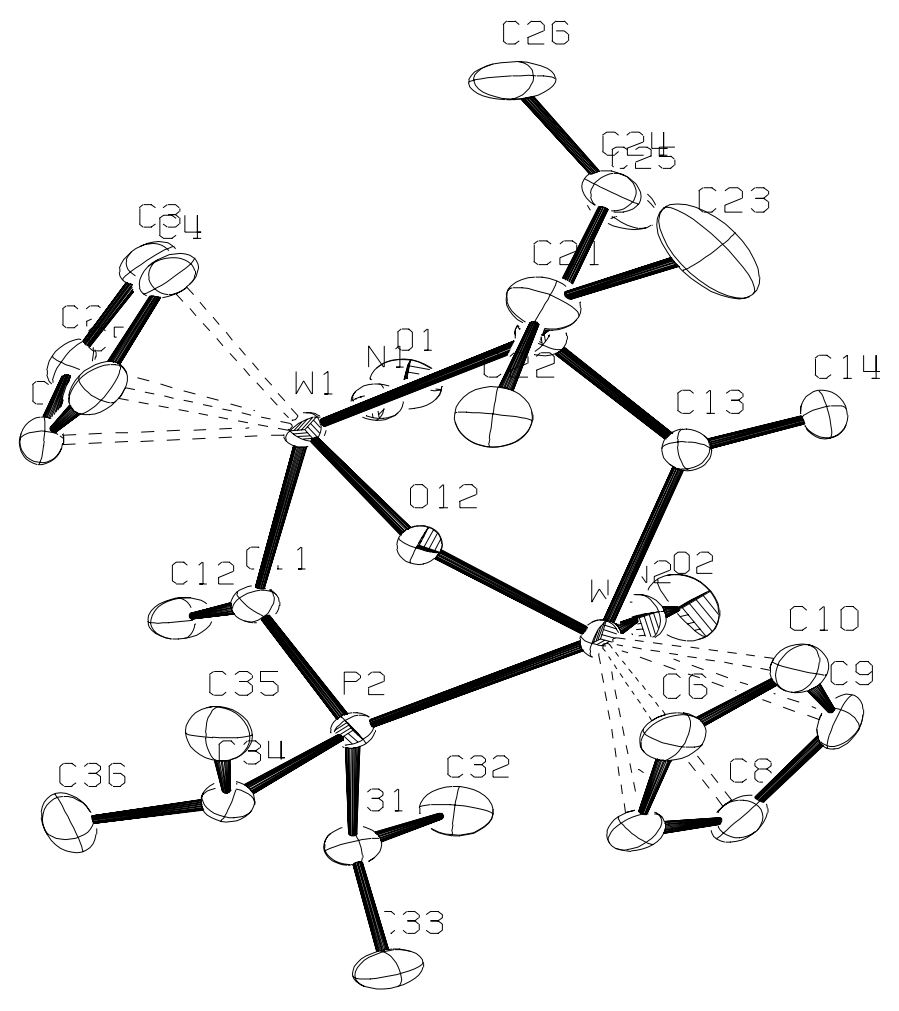


Table 1. Crystal data and structure refinement for $\mathbf{5}$.

Identification code

Empirical formula

Formula weight

Temperature

Wavelength

Crystal system

Space group

Unit cell dimensions

Volume

$\mathrm{Z}$

Density (calculated)

Absorption coefficient

$\mathrm{F}(000)$

Crystal size

Theta range for data collection

Index ranges

Reflections collected

Independent reflections

Completeness to theta $=25.88^{\circ}$

Refinement method

Data / restraints / parameters

Goodness-of-fit on $\mathrm{F}^{2}$

Final $\mathrm{R}$ indices [I $>2 \operatorname{sigma}(\mathrm{I})]$

$\mathrm{R}$ indices (all data)

Largest diff. peak and hole ipa55

C104 H168 N8 O12 P8 W8

3441.02

293(2) K

$0.71073 \AA$

monoclinic

$\mathrm{P} 2{ }_{1} / \mathrm{c}$

$a=10.1365(12) \AA$

$\alpha=90.067(14)^{\circ}$.

$\mathrm{b}=14.4120(16) \AA$

$\beta=109.257(14)^{\circ}$.

$\mathrm{c}=21.374(2) \AA$

$\gamma=89.950(14)^{\circ}$.

2947.8(6) $\AA^{3}$

1

$1.938 \mathrm{Mg} / \mathrm{m}^{3}$

$7.936 \mathrm{~mm}^{-1}$

1656

$0.35 \times 0.27 \times 0.34 \mathrm{~mm}^{3}$

2.40 to $25.88^{\circ}$.

$-12<=\mathrm{h}<=12,-17<=\mathrm{k}<=16,-26<=\mathrm{l}<=26$

20908

$5687[\mathrm{R}(\mathrm{int})=0.0501]$

$99.2 \%$

Full-matrix least-squares on $\mathrm{F}^{2}$

5687 / 0 / 316

1.039

$\mathrm{R} 1=0.0226, \mathrm{wR} 2=0.0580$

$\mathrm{R} 1=0.0273, \mathrm{wR} 2=0.0596$

1.271 and -0.735 e. $\AA^{-3}$ 
Table 2. Atomic coordinates $\left(\times 10^{4}\right)$ and equivalent isotropic displacement parameters $\left(\AA^{2} \times 10^{3}\right)$ for 5. $\mathrm{U}(\mathrm{eq})$ is defined as one third of the trace of the orthogonalized $\mathrm{U}^{\mathrm{ij}}$ tensor.

\begin{tabular}{|c|c|c|c|c|}
\hline & $\mathrm{x}$ & $\mathrm{y}$ & $\mathrm{z}$ & $\mathrm{U}(\mathrm{eq})$ \\
\hline $\mathrm{W}(1)$ & $-102(1)$ & $2723(1)$ & $4375(1)$ & $27(1)$ \\
\hline $\mathrm{N}(1)$ & 2(4) & $3860(3)$ & $4081(2)$ & $36(1)$ \\
\hline $\mathrm{O}(1)$ & $-96(4)$ & $4660(2)$ & $3887(2)$ & $56(1)$ \\
\hline $\mathrm{C}(1)$ & $-1984(5)$ & $2150(4)$ & $4694(3)$ & $47(1)$ \\
\hline $\mathrm{C}(2)$ & $-1852(5)$ & $3120(4)$ & $4800(2)$ & $47(1)$ \\
\hline $\mathrm{C}(3)$ & $-573(6)$ & $3275(4)$ & $5304(2)$ & $49(1)$ \\
\hline $\mathrm{C}(4)$ & $65(6)$ & $2412(4)$ & $5499(2)$ & $49(1)$ \\
\hline$C(5)$ & $-827(6)$ & $1714(4)$ & $5128(2)$ & $49(1)$ \\
\hline $\mathrm{P}(1)$ & $2537(1)$ & $2641(1)$ & $4819(1)$ & $34(1)$ \\
\hline $\mathrm{C}(13)$ & $3042(5)$ & $2505(3)$ & $4085(2)$ & $38(1)$ \\
\hline $\mathrm{C}(14)$ & 4195(6) & $2900(5)$ & 4063(3) & $70(2)$ \\
\hline $\mathrm{C}(21)$ & $3310(6)$ & $1621(4)$ & $5363(3)$ & $52(1)$ \\
\hline $\mathrm{C}(22)$ & $2728(6)$ & $697(4)$ & $5086(3)$ & $57(1)$ \\
\hline $\mathrm{C}(23)$ & 4897(7) & $1612(6)$ & $5556(5)$ & $115(3)$ \\
\hline $\mathrm{C}(24)$ & $3553(5)$ & $3630(4)$ & $5296(2)$ & $48(1)$ \\
\hline $\mathrm{C}(25)$ & $3113(6)$ & $4532(4)$ & 4922(3) & $67(2)$ \\
\hline$C(26)$ & $3413(8)$ & $3717(6)$ & $5986(3)$ & $85(2)$ \\
\hline $\mathrm{O}(12)$ & $692(3)$ & $1692(2)$ & 3991(1) & $28(1)$ \\
\hline $\mathrm{W}(2)$ & 1452(1) & $1810(1)$ & $3245(1)$ & $28(1)$ \\
\hline $\mathrm{N}(2)$ & $1270(4)$ & 2915(3) & $2881(2)$ & $44(1)$ \\
\hline $\mathrm{O}(2)$ & $1289(5)$ & $3658(3)$ & $2605(2)$ & $76(1)$ \\
\hline$C(6)$ & $2327(5)$ & $227(3)$ & $3297(3)$ & $47(1)$ \\
\hline$C(7)$ & $1323(5)$ & $357(3)$ & $2673(2)$ & $46(1)$ \\
\hline $\mathrm{C}(8)$ & $1800(5)$ & $1061(4)$ & $2349(2)$ & $52(1)$ \\
\hline $\mathrm{C}(9)$ & $3101(5)$ & $1368(4)$ & 2771(3) & $57(1)$ \\
\hline$C(10)$ & $3406(5)$ & $848(4)$ & $3363(3)$ & $52(1)$ \\
\hline $\mathrm{P}(2)$ & $-1176(1)$ & $1658(1)$ & 2874(1) & $28(1)$ \\
\hline $\mathrm{C}(11)$ & $-1671(4)$ & 2519(3) & $3384(2)$ & $34(1)$ \\
\hline $\mathrm{C}(12)$ & $-2802(5)$ & $3033(4)$ & $3128(3)$ & $54(1)$ \\
\hline $\mathrm{C}(31)$ & $-2235(5)$ & 1895(3) & $1996(2)$ & $39(1)$ \\
\hline $\mathrm{C}(32)$ & $-1682(7)$ & $2762(4)$ & $1757(3)$ & $59(2)$ \\
\hline $\mathrm{C}(33)$ & $-2276(6)$ & $1085(4)$ & $1534(2)$ & $55(1)$ \\
\hline $\mathrm{C}(34)$ & $-1899(5)$ & $522(3)$ & $3012(2)$ & $38(1)$ \\
\hline $\mathrm{C}(35)$ & $-1104(6)$ & $40(4)$ & $3658(2)$ & $51(1)$ \\
\hline
\end{tabular}


C(36)

$-3433(5)$

580(4)

2938(3)

64(2) 
Table 3. Bond lengths $[\AA]$ and angles $\left[^{\circ}\right]$ for 5 .

\begin{tabular}{|c|c|}
\hline $\mathrm{W}(1)-\mathrm{N}(1)$ & $1.772(4)$ \\
\hline $\mathrm{W}(1)-\mathrm{O}(12)$ & $1.989(2)$ \\
\hline W(1)-C(11) & $2.209(4)$ \\
\hline $\mathrm{W}(1)-\mathrm{C}(2)$ & $2.315(4)$ \\
\hline $\mathrm{W}(1)-\mathrm{C}(3)$ & $2.328(4)$ \\
\hline $\mathrm{W}(1)-\mathrm{C}(1)$ & $2.377(4)$ \\
\hline $\mathrm{W}(1)-\mathrm{C}(4)$ & $2.396(5)$ \\
\hline $\mathrm{W}(1)-\mathrm{C}(5)$ & $2.456(4)$ \\
\hline $\mathrm{W}(1)-\mathrm{P}(1)$ & $2.5289(12)$ \\
\hline $\mathrm{N}(1)-\mathrm{O}(1)$ & $1.219(5)$ \\
\hline $\mathrm{C}(1)-\mathrm{C}(5)$ & $1.383(8)$ \\
\hline $\mathrm{C}(1)-\mathrm{C}(2)$ & $1.416(7)$ \\
\hline $\mathrm{C}(2)-\mathrm{C}(3)$ & $1.404(7)$ \\
\hline$C(3)-C(4)$ & $1.400(7)$ \\
\hline$C(4)-C(5)$ & $1.409(8)$ \\
\hline $\mathrm{P}(1)-\mathrm{C}(13)$ & $1.813(4)$ \\
\hline $\mathrm{P}(1)-\mathrm{C}(24)$ & $1.855(5)$ \\
\hline$P(1)-C(21)$ & $1.878(5)$ \\
\hline $\mathrm{C}(13)-\mathrm{C}(14)$ & $1.315(7)$ \\
\hline$C(13)-W(2)$ & $2.217(5)$ \\
\hline$C(21)-C(22)$ & $1.498(8)$ \\
\hline $\mathrm{C}(21)-\mathrm{C}(23)$ & $1.523(8)$ \\
\hline$C(24)-C(25)$ & $1.515(8)$ \\
\hline $\mathrm{C}(24)-\mathrm{C}(26)$ & $1.531(7)$ \\
\hline $\mathrm{O}(12)-\mathrm{W}(2)$ & $1.995(2)$ \\
\hline $\mathrm{W}(2)-\mathrm{N}(2)$ & $1.755(4)$ \\
\hline $\mathrm{W}(2)-\mathrm{C}(9)$ & $2.310(4)$ \\
\hline $\mathrm{W}(2)-\mathrm{C}(8)$ & $2.323(4)$ \\
\hline $\mathrm{W}(2)-\mathrm{C}(10)$ & $2.361(4)$ \\
\hline $\mathrm{W}(2)-\mathrm{C}(7)$ & $2.406(4)$ \\
\hline $\mathrm{W}(2)-\mathrm{C}(6)$ & $2.437(5)$ \\
\hline $\mathrm{W}(2)-\mathrm{P}(2)$ & $2.5256(11)$ \\
\hline $\mathrm{N}(2)-\mathrm{O}(2)$ & $1.227(5)$ \\
\hline$C(6)-C(10)$ & $1.384(7)$ \\
\hline$C(6)-C(7)$ & $1.398(7)$ \\
\hline$C(7)-C(8)$ & $1.402(7)$ \\
\hline $\mathrm{C}(8)-\mathrm{C}(9)$ & $1.401(8)$ \\
\hline
\end{tabular}




\begin{tabular}{|c|c|}
\hline$C(9)-C(10)$ & $1.415(8)$ \\
\hline $\mathrm{P}(2)-\mathrm{C}(11)$ & $1.825(4)$ \\
\hline $\mathrm{P}(2)-\mathrm{C}(34)$ & $1.857(4)$ \\
\hline $\mathrm{P}(2)-\mathrm{C}(31)$ & $1.861(4)$ \\
\hline$C(11)-C(12)$ & $1.323(6)$ \\
\hline$C(31)-C(33)$ & $1.521(6)$ \\
\hline$C(31)-C(32)$ & $1.526(7)$ \\
\hline$C(34)-C(36)$ & $1.513(6)$ \\
\hline C(34)-C(35) & $1.518(7)$ \\
\hline $\mathrm{N}(1)-\mathrm{W}(1)-\mathrm{O}(12)$ & $117.71(13)$ \\
\hline $\mathrm{N}(1)-\mathrm{W}(1)-\mathrm{C}(11)$ & $83.76(16)$ \\
\hline $\mathrm{O}(12)-\mathrm{W}(1)-\mathrm{C}(11)$ & $76.52(13)$ \\
\hline $\mathrm{N}(1)-\mathrm{W}(1)-\mathrm{C}(2)$ & $93.11(17)$ \\
\hline $\mathrm{O}(12)-\mathrm{W}(1)-\mathrm{C}(2)$ & $144.28(15)$ \\
\hline $\mathrm{C}(11)-\mathrm{W}(1)-\mathrm{C}(2)$ & $90.39(17)$ \\
\hline $\mathrm{N}(1)-\mathrm{W}(1)-\mathrm{C}(3)$ & $92.29(17)$ \\
\hline $\mathrm{O}(12)-\mathrm{W}(1)-\mathrm{C}(3)$ & $146.07(15)$ \\
\hline $\mathrm{C}(11)-\mathrm{W}(1)-\mathrm{C}(3)$ & $125.31(17)$ \\
\hline $\mathrm{C}(2)-\mathrm{W}(1)-\mathrm{C}(3)$ & $35.19(18)$ \\
\hline $\mathrm{N}(1)-\mathrm{W}(1)-\mathrm{C}(1)$ & $124.86(17)$ \\
\hline $\mathrm{O}(12)-\mathrm{W}(1)-\mathrm{C}(1)$ & $109.30(15)$ \\
\hline $\mathrm{C}(11)-\mathrm{W}(1)-\mathrm{C}(1)$ & $80.62(17)$ \\
\hline $\mathrm{C}(2)-\mathrm{W}(1)-\mathrm{C}(1)$ & $35.09(18)$ \\
\hline $\mathrm{C}(3)-\mathrm{W}(1)-\mathrm{C}(1)$ & $57.62(18)$ \\
\hline $\mathrm{N}(1)-\mathrm{W}(1)-\mathrm{C}(4)$ & $122.47(17)$ \\
\hline $\mathrm{O}(12)-\mathrm{W}(1)-\mathrm{C}(4)$ & $111.64(15)$ \\
\hline $\mathrm{C}(11)-\mathrm{W}(1)-\mathrm{C}(4)$ & $136.67(17)$ \\
\hline $\mathrm{C}(2)-\mathrm{W}(1)-\mathrm{C}(4)$ & $57.48(19)$ \\
\hline $\mathrm{C}(3)-\mathrm{W}(1)-\mathrm{C}(4)$ & $34.43(18)$ \\
\hline $\mathrm{C}(1)-\mathrm{W}(1)-\mathrm{C}(4)$ & $56.25(18)$ \\
\hline $\mathrm{N}(1)-\mathrm{W}(1)-\mathrm{C}(5)$ & $147.88(15)$ \\
\hline $\mathrm{O}(12)-\mathrm{W}(1)-\mathrm{C}(5)$ & $94.40(13)$ \\
\hline $\mathrm{C}(11)-\mathrm{W}(1)-\mathrm{C}(5)$ & $105.87(18)$ \\
\hline $\mathrm{C}(2)-\mathrm{W}(1)-\mathrm{C}(5)$ & $56.99(18)$ \\
\hline $\mathrm{C}(3)-\mathrm{W}(1)-\mathrm{C}(5)$ & $56.87(17)$ \\
\hline$C(1)-W(1)-C(5)$ & $33.21(18)$ \\
\hline $\mathrm{C}(4)-\mathrm{W}(1)-\mathrm{C}(5)$ & $33.75(18)$ \\
\hline $\mathrm{N}(1)-\mathrm{W}(1)-\mathrm{P}(1)$ & $89.80(12)$ \\
\hline
\end{tabular}




\begin{tabular}{|c|c|}
\hline $\mathrm{O}(12)-\mathrm{W}(1)-\mathrm{P}(1)$ & $66.30(8)$ \\
\hline $\mathrm{C}(11)-\mathrm{W}(1)-\mathrm{P}(1)$ & $133.72(10)$ \\
\hline $\mathrm{C}(2)-\mathrm{W}(1)-\mathrm{P}(1)$ & $135.80(14)$ \\
\hline $\mathrm{C}(3)-\mathrm{W}(1)-\mathrm{P}(1)$ & $100.65(14)$ \\
\hline $\mathrm{C}(1)-\mathrm{W}(1)-\mathrm{P}(1)$ & $136.56(14)$ \\
\hline $\mathrm{C}(4)-\mathrm{W}(1)-\mathrm{P}(1)$ & $84.21(13)$ \\
\hline $\mathrm{C}(5)-\mathrm{W}(1)-\mathrm{P}(1)$ & $103.58(14)$ \\
\hline $\mathrm{O}(1)-\mathrm{N}(1)-\mathrm{W}(1)$ & $171.6(3)$ \\
\hline $\mathrm{C}(5)-\mathrm{C}(1)-\mathrm{C}(2)$ & 109.1(5) \\
\hline $\mathrm{C}(5)-\mathrm{C}(1)-\mathrm{W}(1)$ & $76.5(3)$ \\
\hline $\mathrm{C}(2)-\mathrm{C}(1)-\mathrm{W}(1)$ & $70.1(2)$ \\
\hline $\mathrm{C}(3)-\mathrm{C}(2)-\mathrm{C}(1)$ & $107.1(5)$ \\
\hline $\mathrm{C}(3)-\mathrm{C}(2)-\mathrm{W}(1)$ & $72.9(2)$ \\
\hline $\mathrm{C}(1)-\mathrm{C}(2)-\mathrm{W}(1)$ & $74.8(2)$ \\
\hline $\mathrm{C}(4)-\mathrm{C}(3)-\mathrm{C}(2)$ & $107.9(5)$ \\
\hline $\mathrm{C}(4)-\mathrm{C}(3)-\mathrm{W}(1)$ & $75.4(3)$ \\
\hline $\mathrm{C}(2)-\mathrm{C}(3)-\mathrm{W}(1)$ & $71.9(2)$ \\
\hline $\mathrm{C}(3)-\mathrm{C}(4)-\mathrm{C}(5)$ & $108.6(5)$ \\
\hline $\mathrm{C}(3)-\mathrm{C}(4)-\mathrm{W}(1)$ & $70.1(3)$ \\
\hline $\mathrm{C}(5)-\mathrm{C}(4)-\mathrm{W}(1)$ & $75.5(3)$ \\
\hline $\mathrm{C}(1)-\mathrm{C}(5)-\mathrm{C}(4)$ & $107.4(5)$ \\
\hline $\mathrm{C}(1)-\mathrm{C}(5)-\mathrm{W}(1)$ & $70.3(2)$ \\
\hline $\mathrm{C}(4)-\mathrm{C}(5)-\mathrm{W}(1)$ & $70.8(3)$ \\
\hline $\mathrm{C}(13)-\mathrm{P}(1)-\mathrm{C}(24)$ & 107.1(2) \\
\hline $\mathrm{C}(13)-\mathrm{P}(1)-\mathrm{C}(21)$ & $105.9(2)$ \\
\hline $\mathrm{C}(24)-\mathrm{P}(1)-\mathrm{C}(21)$ & $102.6(3)$ \\
\hline $\mathrm{C}(13)-\mathrm{P}(1)-\mathrm{W}(1)$ & $104.27(15)$ \\
\hline $\mathrm{C}(24)-\mathrm{P}(1)-\mathrm{W}(1)$ & $119.82(17)$ \\
\hline $\mathrm{C}(21)-\mathrm{P}(1)-\mathrm{W}(1)$ & $116.26(18)$ \\
\hline $\mathrm{C}(14)-\mathrm{C}(13)-\mathrm{P}(1)$ & $119.6(4)$ \\
\hline $\mathrm{C}(14)-\mathrm{C}(13)-\mathrm{W}(2)$ & $125.7(4)$ \\
\hline $\mathrm{P}(1)-\mathrm{C}(13)-\mathrm{W}(2)$ & $114.2(2)$ \\
\hline $\mathrm{C}(22)-\mathrm{C}(21)-\mathrm{C}(23)$ & $110.0(5)$ \\
\hline $\mathrm{C}(22)-\mathrm{C}(21)-\mathrm{P}(1)$ & $114.9(4)$ \\
\hline $\mathrm{C}(23)-\mathrm{C}(21)-\mathrm{P}(1)$ & $111.2(4)$ \\
\hline $\mathrm{C}(25)-\mathrm{C}(24)-\mathrm{C}(26)$ & $109.5(5)$ \\
\hline $\mathrm{C}(25)-\mathrm{C}(24)-\mathrm{P}(1)$ & $110.8(4)$ \\
\hline $\mathrm{C}(26)-\mathrm{C}(24)-\mathrm{P}(1)$ & $112.4(4)$ \\
\hline $\mathrm{W}(1)-\mathrm{O}(12)-\mathrm{W}(2)$ & $125.48(13)$ \\
\hline
\end{tabular}




\begin{tabular}{|c|c|}
\hline $\mathrm{N}(2)-\mathrm{W}(2)-\mathrm{O}(12)$ & $114.92(15)$ \\
\hline $\mathrm{N}(2)-\mathrm{W}(2)-\mathrm{C}(13)$ & $83.76(19)$ \\
\hline $\mathrm{O}(12)-\mathrm{W}(2)-\mathrm{C}(13)$ & $76.17(13)$ \\
\hline $\mathrm{N}(2)-\mathrm{W}(2)-\mathrm{C}(9)$ & $92.3(2)$ \\
\hline $\mathrm{O}(12)-\mathrm{W}(2)-\mathrm{C}(9)$ & $148.50(17)$ \\
\hline $\mathrm{C}(13)-\mathrm{W}(2)-\mathrm{C}(9)$ & $92.33(18)$ \\
\hline $\mathrm{N}(2)-\mathrm{W}(2)-\mathrm{C}(8)$ & $94.5(2)$ \\
\hline $\mathrm{O}(12)-\mathrm{W}(2)-\mathrm{C}(8)$ & $145.36(16)$ \\
\hline $\mathrm{C}(13)-\mathrm{W}(2)-\mathrm{C}(8)$ & $127.53(17)$ \\
\hline $\mathrm{C}(9)-\mathrm{W}(2)-\mathrm{C}(8)$ & $35.21(19)$ \\
\hline $\mathrm{N}(2)-\mathrm{W}(2)-\mathrm{C}(10)$ & $122.79(19)$ \\
\hline $\mathrm{O}(12)-\mathrm{W}(2)-\mathrm{C}(10)$ & $113.26(16)$ \\
\hline $\mathrm{C}(13)-\mathrm{W}(2)-\mathrm{C}(10)$ & $79.51(17)$ \\
\hline $\mathrm{C}(9)-\mathrm{W}(2)-\mathrm{C}(10)$ & $35.24(19)$ \\
\hline $\mathrm{C}(8)-\mathrm{W}(2)-\mathrm{C}(10)$ & $57.73(19)$ \\
\hline $\mathrm{N}(2)-\mathrm{W}(2)-\mathrm{C}(7)$ & $125.73(18)$ \\
\hline $\mathrm{O}(12)-\mathrm{W}(2)-\mathrm{C}(7)$ & $111.14(14)$ \\
\hline $\mathrm{C}(13)-\mathrm{W}(2)-\mathrm{C}(7)$ & 135.02(17) \\
\hline $\mathrm{C}(9)-\mathrm{W}(2)-\mathrm{C}(7)$ & $57.60(19)$ \\
\hline $\mathrm{C}(8)-\mathrm{W}(2)-\mathrm{C}(7)$ & $34.43(18)$ \\
\hline$C(10)-W(2)-C(7)$ & $56.41(18)$ \\
\hline $\mathrm{N}(2)-\mathrm{W}(2)-\mathrm{C}(6)$ & $148.55(17)$ \\
\hline $\mathrm{O}(12)-\mathrm{W}(2)-\mathrm{C}(6)$ & $96.48(14)$ \\
\hline $\mathrm{C}(13)-\mathrm{W}(2)-\mathrm{C}(6)$ & $103.23(17)$ \\
\hline $\mathrm{C}(9)-\mathrm{W}(2)-\mathrm{C}(6)$ & $57.18(19)$ \\
\hline $\mathrm{C}(8)-\mathrm{W}(2)-\mathrm{C}(6)$ & $56.76(18)$ \\
\hline$C(10)-W(2)-C(6)$ & $33.48(17)$ \\
\hline$C(7)-W(2)-C(6)$ & $33.56(17)$ \\
\hline $\mathrm{N}(2)-\mathrm{W}(2)-\mathrm{P}(2)$ & $89.71(13)$ \\
\hline $\mathrm{O}(12)-\mathrm{W}(2)-\mathrm{P}(2)$ & $66.28(8)$ \\
\hline $\mathrm{C}(13)-\mathrm{W}(2)-\mathrm{P}(2)$ & 134.71(11) \\
\hline $\mathrm{C}(9)-\mathrm{W}(2)-\mathrm{P}(2)$ & $132.78(15)$ \\
\hline $\mathrm{C}(8)-\mathrm{W}(2)-\mathrm{P}(2)$ & $97.60(13)$ \\
\hline $\mathrm{C}(10)-\mathrm{W}(2)-\mathrm{P}(2)$ & $137.96(14)$ \\
\hline $\mathrm{C}(7)-\mathrm{W}(2)-\mathrm{P}(2)$ & $83.67(12)$ \\
\hline $\mathrm{C}(6)-\mathrm{W}(2)-\mathrm{P}(2)$ & $105.23(12)$ \\
\hline $\mathrm{O}(2)-\mathrm{N}(2)-\mathrm{W}(2)$ & $171.9(4)$ \\
\hline$C(10)-C(6)-C(7)$ & $108.1(5)$ \\
\hline $\mathrm{C}(10)-\mathrm{C}(6)-\mathrm{W}(2)$ & $70.3(3)$ \\
\hline
\end{tabular}




\begin{tabular}{|c|c|}
\hline $\mathrm{C}(7)-\mathrm{C}(6)-\mathrm{W}(2)$ & $72.0(3)$ \\
\hline$C(6)-C(7)-C(8)$ & $107.9(5)$ \\
\hline $\mathrm{C}(6)-\mathrm{C}(7)-\mathrm{W}(2)$ & $74.5(3)$ \\
\hline $\mathrm{C}(8)-\mathrm{C}(7)-\mathrm{W}(2)$ & $69.5(3)$ \\
\hline $\mathrm{C}(9)-\mathrm{C}(8)-\mathrm{C}(7)$ & $108.4(5)$ \\
\hline $\mathrm{C}(9)-\mathrm{C}(8)-\mathrm{W}(2)$ & $71.9(3)$ \\
\hline $\mathrm{C}(7)-\mathrm{C}(8)-\mathrm{W}(2)$ & $76.0(2)$ \\
\hline $\mathrm{C}(8)-\mathrm{C}(9)-\mathrm{C}(10)$ & $106.8(5)$ \\
\hline $\mathrm{C}(8)-\mathrm{C}(9)-\mathrm{W}(2)$ & $72.9(2)$ \\
\hline $\mathrm{C}(10)-\mathrm{C}(9)-\mathrm{W}(2)$ & $74.4(2)$ \\
\hline $\mathrm{C}(6)-\mathrm{C}(10)-\mathrm{C}(9)$ & $108.7(5)$ \\
\hline $\mathrm{C}(6)-\mathrm{C}(10)-\mathrm{W}(2)$ & $76.3(3)$ \\
\hline $\mathrm{C}(9)-\mathrm{C}(10)-\mathrm{W}(2)$ & $70.4(3)$ \\
\hline $\mathrm{C}(11)-\mathrm{P}(2)-\mathrm{C}(34)$ & 107.67(19) \\
\hline $\mathrm{C}(11)-\mathrm{P}(2)-\mathrm{C}(31)$ & $107.2(2)$ \\
\hline $\mathrm{C}(34)-\mathrm{P}(2)-\mathrm{C}(31)$ & $101.3(2)$ \\
\hline $\mathrm{C}(11)-\mathrm{P}(2)-\mathrm{W}(2)$ & $102.85(14)$ \\
\hline $\mathrm{C}(34)-\mathrm{P}(2)-\mathrm{W}(2)$ & $117.27(15)$ \\
\hline $\mathrm{C}(31)-\mathrm{P}(2)-\mathrm{W}(2)$ & $119.86(15)$ \\
\hline $\mathrm{C}(12)-\mathrm{C}(11)-\mathrm{P}(2)$ & $120.3(4)$ \\
\hline $\mathrm{C}(12)-\mathrm{C}(11)-\mathrm{W}(1)$ & $125.0(3)$ \\
\hline $\mathrm{P}(2)-\mathrm{C}(11)-\mathrm{W}(1)$ & $114.2(2)$ \\
\hline $\mathrm{C}(33)-\mathrm{C}(31)-\mathrm{C}(32)$ & $110.2(4)$ \\
\hline $\mathrm{C}(33)-\mathrm{C}(31)-\mathrm{P}(2)$ & $113.4(3)$ \\
\hline $\mathrm{C}(32)-\mathrm{C}(31)-\mathrm{P}(2)$ & $109.6(3)$ \\
\hline $\mathrm{C}(36)-\mathrm{C}(34)-\mathrm{C}(35)$ & $109.8(4)$ \\
\hline $\mathrm{C}(36)-\mathrm{C}(34)-\mathrm{P}(2)$ & $112.4(4)$ \\
\hline $\mathrm{C}(35)-\mathrm{C}(34)-\mathrm{P}(2)$ & $115.4(3)$ \\
\hline
\end{tabular}

Symmetry transformations used to generate equivalent atoms: 
Table 4. Anisotropic displacement parameters $\left(\AA^{2} \times 10^{3}\right)$ for $\mathbf{5}$. The anisotropic displacement factor exponent takes the form: $-2 \pi^{2}\left[h^{2} a^{* 2} U^{11}+\ldots+2 h k a^{*} b^{*} U^{12}\right]$

\begin{tabular}{|c|c|c|c|c|c|c|}
\hline & $\mathrm{U}^{11}$ & $\mathrm{U}^{22}$ & $\mathrm{U}^{33}$ & $\mathrm{U}^{23}$ & $\mathrm{U}^{13}$ & $\mathrm{U}^{12}$ \\
\hline $\mathrm{W}(1)$ & $30(1)$ & $25(1)$ & $28(1)$ & $-2(1)$ & $12(1)$ & $1(1)$ \\
\hline $\mathrm{N}(1)$ & $38(2)$ & $34(2)$ & $35(2)$ & $-3(2)$ & $9(2)$ & $1(2)$ \\
\hline $\mathrm{O}(1)$ & $76(3)$ & $25(2)$ & $61(2)$ & $11(2)$ & $14(2)$ & $2(2)$ \\
\hline $\mathrm{C}(1)$ & $43(3)$ & $54(3)$ & $54(3)$ & $-8(2)$ & $30(2)$ & $-12(2)$ \\
\hline $\mathrm{C}(2)$ & $48(3)$ & $54(3)$ & $51(3)$ & $-5(2)$ & $32(2)$ & $8(2)$ \\
\hline $\mathrm{C}(3)$ & $65(3)$ & $50(3)$ & $42(3)$ & $-10(2)$ & $31(3)$ & $-3(2)$ \\
\hline $\mathrm{C}(4)$ & $60(3)$ & $59(3)$ & $35(2)$ & $-3(2)$ & $26(2)$ & $0(3)$ \\
\hline $\mathrm{C}(5)$ & $70(3)$ & $39(3)$ & $50(3)$ & $5(2)$ & $39(3)$ & $2(2)$ \\
\hline $\mathrm{P}(1)$ & $31(1)$ & $32(1)$ & $35(1)$ & $-1(1)$ & $6(1)$ & $0(1)$ \\
\hline $\mathrm{C}(13)$ & $33(2)$ & $40(3)$ & $42(2)$ & $-5(2)$ & $13(2)$ & $0(2)$ \\
\hline $\mathrm{C}(14)$ & $46(3)$ & $95(5)$ & $81(4)$ & $-39(4)$ & $37(3)$ & $-29(3)$ \\
\hline $\mathrm{C}(21)$ & $48(3)$ & $48(3)$ & $49(3)$ & $11(2)$ & $-2(2)$ & $10(2)$ \\
\hline $\mathrm{C}(22)$ & $65(3)$ & $44(3)$ & $56(3)$ & $17(2)$ & 14(3) & 11(3) \\
\hline $\mathrm{C}(23)$ & $50(4)$ & $73(5)$ & $180(9)$ & $36(6)$ & $-19(5)$ & $14(4)$ \\
\hline$C(24)$ & $38(2)$ & $53(3)$ & $47(3)$ & $-11(2)$ & $5(2)$ & $-6(2)$ \\
\hline$C(25)$ & $59(3)$ & $39(3)$ & $90(4)$ & $-15(3)$ & $8(3)$ & $-9(3)$ \\
\hline$C(26)$ & $103(5)$ & $96(6)$ & $51(3)$ & $-38(4)$ & $19(4)$ & $-29(4)$ \\
\hline $\mathrm{O}(12)$ & $30(1)$ & $25(1)$ & $30(1)$ & $2(1)$ & $12(1)$ & $2(1)$ \\
\hline $\mathrm{W}(2)$ & $27(1)$ & $27(1)$ & $32(1)$ & $0(1)$ & $14(1)$ & $2(1)$ \\
\hline $\mathrm{N}(2)$ & $45(2)$ & $43(2)$ & $48(2)$ & $6(2)$ & $17(2)$ & $-3(2)$ \\
\hline $\mathrm{O}(2)$ & $87(3)$ & $48(2)$ & $92(3)$ & $35(2)$ & $27(3)$ & $-9(2)$ \\
\hline $\mathrm{C}(6)$ & $52(3)$ & $33(3)$ & $57(3)$ & $-9(2)$ & $19(2)$ & $13(2)$ \\
\hline $\mathrm{C}(7)$ & $46(3)$ & $40(3)$ & $54(3)$ & $-18(2)$ & $21(2)$ & $6(2)$ \\
\hline $\mathrm{C}(8)$ & $52(3)$ & $70(4)$ & $42(2)$ & $-8(2)$ & $24(2)$ & $9(3)$ \\
\hline $\mathrm{C}(9)$ & $47(3)$ & $68(4)$ & $70(3)$ & $-12(3)$ & $40(3)$ & $4(3)$ \\
\hline$C(10)$ & $37(2)$ & $57(3)$ & $62(3)$ & $-17(3)$ & $15(2)$ & $14(2)$ \\
\hline $\mathrm{P}(2)$ & $29(1)$ & $26(1)$ & $28(1)$ & $-1(1)$ & 11(1) & $3(1)$ \\
\hline $\mathrm{C}(11)$ & $33(2)$ & $32(2)$ & $36(2)$ & $-4(2)$ & $11(2)$ & $2(2)$ \\
\hline $\mathrm{C}(12)$ & $43(3)$ & $64(4)$ & $47(3)$ & $-13(3)$ & $4(2)$ & $21(3)$ \\
\hline $\mathrm{C}(31)$ & $40(2)$ & $39(3)$ & $33(2)$ & $2(2)$ & $4(2)$ & $10(2)$ \\
\hline$C(32)$ & $72(4)$ & $52(4)$ & $42(3)$ & $17(2)$ & $3(3)$ & $9(3)$ \\
\hline $\mathrm{C}(33)$ & $65(3)$ & $61(4)$ & $34(2)$ & $-9(2)$ & $9(2)$ & $7(3)$ \\
\hline$C(34)$ & $43(2)$ & $32(2)$ & $42(2)$ & $-5(2)$ & $17(2)$ & $-3(2)$ \\
\hline$C(35)$ & $66(3)$ & $36(3)$ & $53(3)$ & $11(2)$ & $23(3)$ & $-10(2)$ \\
\hline
\end{tabular}


C(36)

45(3)

61(4)

90(4)

$-2(3)$

28(3)

$-12(3)$ 
